\title{
STRUCTURALLY STABLE CONFIGURATIONS OF LINES OF MEAN CURVATURE AND UMBILIC POINTS ON SURFACES IMMERSED IN $\mathbb{R}^{3}$
}

\author{
Ronaldo Garcia And Jorge Sotomayor
}

\begin{abstract}
In this paper we study the pairs of orthogonal foliations on oriented surfaces immersed in $\mathbb{R}^{3}$ whose singularities and leaves are, respectively, the umbilic points and the lines of normal mean curvature of the immersion. Along these lines the immersions bend in $\mathbb{R}^{3}$ according to their normal mean curvature. By analogy with the closely related Principal Curvature Configurations studied in $[\mathbf{S}-\mathbf{G}]$, [GS2], whose lines produce the extremal normal curvature for the immersion, the pair of foliations by lines of normal mean curvature and umbilics, assembled together, are called Mean Curvature Configurations. This paper studies the stable and generic cases of umbilic points and mean curvature cycles, with their Poincaré map. This provides two of the essential local ingredients to establish sufficient conditions for mean curvature structural stability, the analog of principal curvature structural stability, [S-G], [GS2].
\end{abstract}

\section{Introduction}

In this paper are studied the Mean Curvature Configurations associated to immersions of an oriented surface into $\mathbb{R}^{3}$. They consist on the Umbilic Points and the Lines of Normal Mean Curvature of the immersion. These lines are characterized by the condition that along them the immersions bend according to their Normal Mean Curvature.

There are many classical pictorial examples and analytic properties of Principal Configurations. Such are the cases of surfaces which are quadratic, of revolution, normally translated, triply orthogonal and inverted,

2000 Mathematics Subject Classification. 53A07, 53A05.

Key words. Umbilic point, mean curvature configuration, lines of mean curvature. The first author was partially supported by CNPq and FAPESP, Grant 99/08487-7. The second author was partially supported by CNPq. This work was done under the project PRONEX/FINEP/MCT - Conv. 76.97.1080.00 - Teoria Qualitativa das Equações Diferenciais Ordinárias. 
among other types. However, the Mean Curvature Configurations do not seem to have been considered before, even for quadrics. An exception is the classical result that on Minimal Surfaces it is given by the Configuration of Asymptotic Lines [St]. In order to illustrate the difference, in Section 5 will be studied the specific examples of ellipsoids and tori, showing that in these cases where the principal lines are trivially periodic, the mean curvature ones may be dense.

One of the sources of interest for this work is the formal analogy with previous work on Principal Configurations [S-G], [GS1]-[GS3], dealing with the umbilic points and integral foliations of tangent principal direction fields along which the immersions bend producing the extremal (principal) normal curvature. In fact, by Euler's Formula, [St], the directions of extremal (i.e. principal) geodesic torsion coincide with those of normal mean curvature.

Also, problems of singularities and global analysis as the Carathéodory conjecture about the number of umbilics on smooth ovaloids is a source for the study of mean curvature lines and principal lines, $[\mathbf{G u}]$, [Ga-M-F-R], [SX1] and [SX2].

It will clear that the specific results suggested by the formal analogy between the Mean and Principal Configurations, when formulated precisely, need to be proved again. When necessary, this will be done here. See for instance Section 3 where the mean curvature separatrix structures at umbilic points, analogous to the Darbouxian principal curvature configurations $[\mathbf{G S 2}]$, has been established. See also $[\mathbf{D a}]$ and [B-F]. There seems to be no general formal argument, a sort of functor, which enables to establish a result in one setting as a corollary of the one to which it is linked by the analogy. However, the methods used for the proofs have some expected similarity, coming from the analysis of quadratic differential equations which govern both Mean and Principal Curvature Lines on a surface.

The ideas formulated above go beyond the formal analogy. This is better explained in terms of the Axial Configuration and the Ellipse of Curvature of immersions into $\mathbb{R}^{4},[\mathbf{L i t}]$. In fact the large axis of the Ellipse of Curvature gives rise naturally to the Principal Curvature Configurations and the Small Axis leads to the Mean Curvature Configurations, as established in [Ga-So1]. See also [G-G-T-G].

Sufficient conditions are provided here to extend to the present Mean Curvature setting the Theorems on Structural Stability for Principal Configurations proved in [S-G], [GS2] and for Axial Structural Stability [Ga-So1]. 
Two local cases are essential for this extension: the umbilic points with the determination of their mean curvature separatrix structure and the mean curvature cycles with the calculation of the derivative of the Poincaré map. Both are treated in detail here. This will establish that, in this aspect, the parallel with the conditions for principal structural stability is remarkable.

This paper is organized as follows:

Section 2 is devoted to the general study of the differential equations of Mean Curvature Lines. The precise definition of Mean Curvature Configuration and Mean Curvature Structural Stability will be given at the end of this section.

In Section 3 the equation of lines of mean curvature is written in a Monge chart. The umbilic stability condition is explicitly stated in terms of the coefficients of the third order jet of the function which represents the immersion in a Monge chart. The local mean curvature separatrix configurations at stable umbilics is established for $C^{4}$ immersions.

In Section 4 the derivative of first return Poincaré map along a mean curvature cycle is established. It consists of an integral expression involving the curvature functions along the cycle.

Section 5 presents examples of Mean Curvature Configurations on the Ellipsoid and the Torus.

In Section 6 the results presented in Sections 3 and 4 are put together to provide sufficient conditions for Mean Curvature Structural Stability.

Section 7 contains the study of the density of Mean Curvature Structurally Stable immersions.

In a sense the present work is intermediate between the studies of Principal and Axial configurations of lines of curvature on a surface. In the forthcoming paper [Ga-So2] the results about Mean and Principal Curvature Structurally Stable Configurations for surfaces immersed into $\mathbb{R}^{3}$ will be put together as essential pieces to provide a class of Structurally Stable Axial Configurations of surfaces immersed into $\mathbb{R}^{4}$. This is achieved by means of a suitable deformation of immersions from $\mathbb{R}^{3}$ into $\mathbb{R}^{4}$. In this paper this is only illustrated in the case of immersions of the Torus free of umbilics. The general problem, proposed in [Ga-So1], will be dealt with in $[\mathbf{G a - S o 2}]$.

Section 8 is devoted to explaining better this point.

\section{Differential equation of mean curvature lines}

Let $\alpha: \mathbb{M}^{2} \rightarrow \mathbb{R}^{3}$ be a $C^{r}, r \geq 4$, immersion of an oriented smooth surface $\mathbb{M}$ into $\mathbb{R}^{3}$. This last space is oriented by a once for all fixed 
orientation and endowed with the Euclidean inner product $\langle$,$\rangle . Let N$ be a vector field orthonormal to $\alpha$. Assume that $(u, v)$ is a positive chart and that $\left\{\alpha_{u}, \alpha_{v}, N\right\}$ is a positive frame in $\mathbb{R}^{3}$.

In the chart $(u, v)$, the first fundamental form of an immersion $\alpha$ is given by: $I_{\alpha}=\langle D \alpha, D \alpha\rangle=E d u^{2}+2 F d u d v+G d v^{2}$, with $E=\left\langle\alpha_{u}, \alpha_{u}\right\rangle$, $F=\left\langle\alpha_{u}, \alpha_{v}\right\rangle, G=\left\langle\alpha_{v}, \alpha_{v}\right\rangle$.

The second fundamental form is given by:

$$
I I_{\alpha}=\left\langle N, D^{2} \alpha\right\rangle=e d u^{2}+2 f d u d v+g d v^{2} .
$$

The normal curvature at a point $p$ in a tangent direction $t=[d u: d v]$ is given by:

$$
k_{n}=k_{n}(p)=\frac{I I_{\alpha}(t, t)}{I_{\alpha}(t, t)} .
$$

The geodesic torsion at a point $p$ in a tangent direction $[d u: d v]$ is given by:

$$
\tau_{g}=\frac{(F g-G f) d v^{2}+(E g-G e) d u d v+(E f-F e) d u^{2}}{\left(E G-F^{2}\right)^{\frac{3}{2}}\left(E d u^{2}+2 F d u d v+G d v^{2}\right)} .
$$

The lines of mean curvature are defined by the condition of having normal curvature equal to the mean curvature, i.e., $k_{n}=\mathcal{H}$. Therefore the pertinent differential equation is given by:

$$
\frac{e d u^{2}+2 f d u d v+g d v^{2}}{E d u^{2}+2 F d u d v+G d v^{2}}=\frac{E g+e G-2 f F}{2\left(E G-F^{2}\right)}=\mathcal{H} .
$$

Or equivalently by

$$
\begin{array}{r}
{\left[g\left(E G-2 F^{2}\right)+2 f F G-e G^{2}\right] d v^{2}+[4 f E G-2 g E F-2 e F G] d u d v} \\
+\left[e\left(E G-2 F^{2}\right)+2 f E F-g E^{2}\right] d u^{2}=0 .
\end{array}
$$

This is equivalent to assert that these lines make extremal the geodesic torsion. In fact, $\tau_{g}= \pm \sqrt{\mathcal{H}^{2}-\mathcal{K}}$ is the extremal value of $\tau_{g}=$ $\sqrt{\mathcal{H}^{2}-\mathcal{K}} \cos \theta \sin \theta$, attained in a mean curvature direction $\theta=\frac{\pi}{4}[\mathbf{S t}]$.

As in the case of principal lines, the two mean curvature directions defined by the equation above are orthogonal with respect to the induced metric. This is immediate by the extremal property of the geodesic torsion.

In a minimal surface mean curvature lines coincide with the asymptotic lines $[\mathbf{S t}]$.

Remark 2.1. The differential equation of mean curvature lines can also be written as

$$
\operatorname{Jac}\left(\operatorname{Jac}\left(I I_{\alpha}, I_{\alpha}\right), I_{\alpha}\right)=0 .
$$


In Monge chart $(u, v, h(u, v))$ the differential equation of lines of mean curvature is given by:

$$
\begin{aligned}
& {\left[h_{v v}\left(1+h_{u}^{2}+h_{v}^{2}-h_{u}^{2} h_{v}^{2}\right)+2 h_{u v} h_{u} h_{v}\left(1+h_{v}^{2}\right)-h_{u u}\left(1+h_{v}^{2}\right)^{2}\right] d v^{2} } \\
+ & {\left[4 h_{u v}\left(1+h_{u}^{2}\right)\left(1+h_{v}^{2}\right)-2 h_{v v} h_{u} h_{v}\left(1+h_{u}^{2}\right)-2 h_{u u} h_{u} h_{v}\left(1+h_{v}^{2}\right)\right] d u d v } \\
+ & {\left[h_{u u}\left(1+h_{u}^{2}+h_{v}^{2}-h_{u}^{2} h_{v}^{2}\right)+2 h_{u v} h_{u} h_{v}\left(1+h_{u}^{2}\right)-h_{v v}\left(1+h_{u}^{2}\right)^{2}\right] d u^{2}=0 . }
\end{aligned}
$$

The developments above allow us to organize the lines of mean curvature of immersions into the mean curvature configuration, as follows:

Through every point $p \in \mathbb{M} \backslash \mathcal{U}_{\alpha}$, where $\mathcal{U}_{\alpha}$ is the umbilic set of $\alpha$, pass two orthogonal mean curvature lines of $\alpha$. Under the orientability hypothesis imposed on $\mathbb{M}$, the mean curvature lines define two foliations $\mathbb{Q}_{\alpha, 1}$, called the minimal mean curvature foliation, along which the geodesic torsion is minimal (i.e. $\tau_{g}=-\sqrt{\mathcal{H}^{2}-\mathcal{K}}$ ), and $\mathbb{Q}_{\alpha, 2}$, called the maximal mean curvature foliations, along which the geodesic torsion is maximal (i.e. $\tau_{g}=+\sqrt{\mathcal{H}^{2}-\mathcal{K}}$ ). These foliations are singular at the umbilic points of $\alpha$. The triple $\mathbb{Q}_{\alpha}=\left\{\mathcal{U}_{\alpha}, \mathbb{Q}_{\alpha, 1}, \mathbb{Q}_{\alpha, 2}\right\}$ is called the mean curvature configuration of $\alpha$.

An immersion $\alpha$ is said $C^{s}$-local mean curvature structurally stable at a compact set $K \subset \mathbb{M}$ if for any sequence of immersions $\alpha_{n}$ converging to $\alpha$ together with its first $s$ derivatives in a compact neighborhood $V_{K}$ of $K$ there is a sequence of compact subsets $K_{n}$ and a sequence of homeomorphisms mapping $K$ to $K_{n}$ converging to the identity of $\mathbb{M}$ such that on $V_{K}$ it maps arcs of the mean curvature foliations $\mathbb{Q}_{\alpha, i}$ to $\operatorname{arcs}$ of $\mathbb{Q}_{\alpha_{n}, i}$ for $i=1,2$.

An immersion $\alpha$ is said $C^{s}$-mean curvature structurally stable if the compact $K$ above is $\mathbb{M}$.

In Sections 3 and 4 will be characterized the umbilics and mean curvature cycles which are local mean curvature structurally stable. Sufficient conditions for immersions to be mean curvature structurally stable are given in Section 6.

\section{Mean curvature lines near umbilic points}

Let 0 be an umbilic point of a $C^{4}$ immersion $\alpha$ parametrized in a Monge chart $(x, y)$ by $\alpha(x, y)=(x, y, h(x, y))$, where

$$
h(x, y)=\frac{k}{2}\left(x^{2}+y^{2}\right)+\frac{a}{6} x^{3}+\frac{b}{2} x y^{2}+\frac{c}{6} y^{3}+O(4) .
$$


The differential equation of principal curvature lines is given by:

$$
-\left[b y+P_{1}\right] d y^{2}+\left[(b-a) x+c y+P_{2}\right] d x d y+\left[b y+P_{3}\right] d x^{2}=0
$$

and the differential equation of the mean curvature lines is given by:

$$
\begin{aligned}
{[(b-a) x+c y} & \left.+M_{1}\right] d y^{2} \\
+ & {\left[4 b y+M_{2}\right] d x d y-\left[(b-a) x+c y+M_{3}\right] d x^{2}=0 }
\end{aligned}
$$

where $M_{i}$ and $P_{i}, i=1,2,3$, represent functions of order $O\left(\left(x^{2}+y^{2}\right)\right)$.

Let

$$
\begin{aligned}
\Delta_{H} & =4 c^{2}(2 a-b)^{2}-\left[3 c^{2}+(a-5 b)^{2}\right]\left[3(a-5 b)(a-b)+c^{2}\right] \\
\Delta_{P} & =4 b(a-2 b)^{3}-c^{2}(a-2 b)^{2} .
\end{aligned}
$$

As a starting point, recall the behavior of principal lines near Darbouxian umbilics in the following proposition.

Proposition 3.1 ([S-G], $[\mathbf{G S 2}])$. Under the conditions above suppose that the transversality condition $T=b(b-a) \neq 0$ holds and consider the following situations:

Di) $\Delta_{P}>0$.

$\left.D_{2}\right) \Delta_{P}<0$ and $\frac{a}{b}>1$.

$\left.D_{3}\right) \frac{a}{b}<1$.

Then each principal foliation has in a neighborhood of 0 , one hyperbolic sector in the $D_{1}$ case, one parabolic and one hyperbolic sector in $D_{2}$ case and three hyperbolic sectors in the case $D_{3}$. The umbilics are called Darbouxians of types $D_{1}, D_{2}$ and $D_{3}$.

Proposition 3.2. Suppose that the transversality condition $T=b(b-$ a) $\neq 0$ holds and consider the following situations:

$\left.H_{1}\right) \Delta_{H}>0$.

$\left.H_{2}\right) \Delta_{H}<0$ and $\frac{a}{b}>1$.

$\left.H_{3}\right) \frac{a}{b}<1$.

Then each mean curvature foliation has in a neighborhood of 0 , one hyperbolic sector in the $H_{1}$ case, one parabolic and one hyperbolic sector in $\mathrm{H}_{2}$ case and three hyperbolic sectors in the case $\mathrm{H}_{3}$. These umbilic points are denoted by $H_{1}, H_{2}$ and $H_{3}$.

The mean curvature configuration $\mathbb{Q}_{\alpha}$ near an umbilic point of type $H_{i}$ has a local behavior as shown in Figure 3.1. 


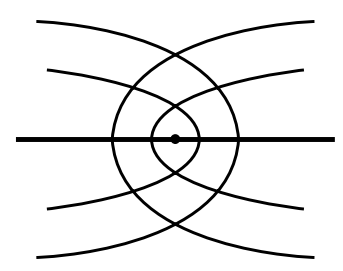

$H_{1}$

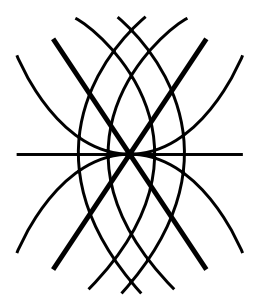

$\mathrm{H}_{2}$

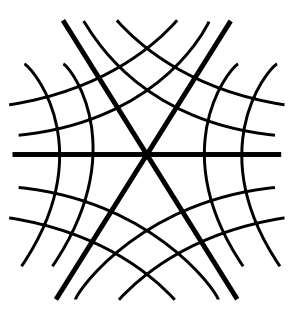

$\mathrm{H}_{3}$

Figure 3.1. Mean curvature lines near the umbilic points $H_{i}$.

Remark 3.1. In the propositions above in $D_{3}$ case (resp. $H_{3}$ case), the condition $\Delta_{P}<0$ (resp. $\left.\Delta_{H}<0\right)$ is automatically satisfied with $\frac{a}{b}<1$.

Proof of Proposition 3.2: The proof is inspired on that of Proposition 3.1, for the case of Darbouxian umbilic points; see [B-F] and [GS2]. Consider the implicit surface

$$
\begin{aligned}
\mathcal{G}(x, y, p)=\left[(b-a) x+c y+M_{1}(2)\right] p^{2} \\
+\left[4 b y+M_{2}(2)\right] p-\left[(b-a) x+c y+M_{3}(2)\right]=0,
\end{aligned}
$$

which, under the transversality condition $T=(b-a) b \neq 0$, is regular near the projective line represented by the $p$-axis i.e. $x=0, y=0$.

In what follows it will be supposed, without loss of generality, that $\frac{c}{b}>0$. The mean curvature lines in the chart $(x, y)$ are the projections by $\pi(x, y, p)=(x, y)$ of the integral curves of the Lie-Cartan line field $X$ given locally by:

$$
X=\mathcal{G}_{p} \frac{\partial}{\partial x}+p \mathcal{G}_{p} \frac{\partial}{\partial y}-\left(\mathcal{G}_{x}+p \mathcal{G}_{y}\right) \frac{\partial}{\partial p} .
$$


The projection $\pi$ is a double regular covering outside the projective line and it is singular only along the projective line.

By the transversality condition $b(b-a) \neq 0$, the singular points of $X$ are contained in the projective line and are given by $(0,0, p)$, where $p$ are the roots of the cubic polynomial

$$
R(p)=\mathcal{G}_{x}+\left.p \mathcal{G}_{y}\right|_{(x, y)=(0,0)}=c p^{3}+(5 b-a) p^{2}-c p+a-b .
$$

The discriminant of the polynomial $R$ is precisely $\Delta_{H}$, defined in equation (3.3).

There are the three cases to consider.

Case 1: $\frac{a}{b}<1$. Then the polynomial $R$ has three real roots, $r_{1} \in(0,1)$, $r_{2} \in(-1,0)$ and $r_{3} \in(-\infty,-1)$. This can be seen intersecting the graphs of the curves $l(p)=\frac{c}{b}\left(p^{3}-p\right)$ and $m(p)=\left(\frac{a}{b}-5\right) p^{2}+1-\frac{a}{b}$.

The linear part of $X$ at a singular point $(0,0, p)$ is given by:

$$
D X(0,0, p)=\left(\begin{array}{ccc}
2 p(b-a) & 4 b+2 c\left(p^{2}-1\right) & 0 \\
2(b-a) p^{2} & \left(4 b+2 c\left(p^{2}-1\right)\right) p & 0 \\
* & * & -R^{\prime}(p)
\end{array}\right) .
$$

Therefore the non zero eigenvalues of $D X(0,0, p)$ are given by:

$$
\lambda_{1}(p)=2(b-a) p+\left(4 b+2 c\left(p^{2}-1\right)\right) p \text { and } \lambda_{2}(p)=-R^{\prime}(p) .
$$

Direct calculation shows that $\left.R\right|_{\left\{\lambda_{1}=0\right\}}=2 b p+a-b$ and $\left.\lambda_{1}\right|_{\{R=0\}}=$ $-\frac{4 b p\left(p^{2}+1\right)}{p^{2}-1}$. Then the two polynomials $R(p)$ and $\lambda_{1}(p)$ have no common roots. So, it is obtained that $\lambda_{1}\left(r_{3}\right)>0, \lambda_{1}\left(r_{2}\right)<0$ and $\lambda_{1}\left(r_{3}\right)>0$.

As we are assuming that $\frac{c}{b}>0$ and $\frac{a}{b}<1$ it follows that $\lambda_{2}\left(r_{3}\right)<0$, $\lambda_{2}\left(r_{2}\right)>0$ and $\lambda_{2}\left(r_{3}\right)<0$.

Therefore, $\lambda_{1}\left(r_{i}\right) \lambda_{2}\left(r_{i}\right)<0$ for $i=1,2,3$. The three singular points are hyperbolic saddles. This ends the proof of the $H_{3}$ case.

Case 2: $\frac{a}{b}>1$ and $\Delta_{H}<0$. Then the polynomial $R(p)$ has three real roots and these are located as follows: $r_{1}, r_{2} \in(0,1), r_{2}<r_{1}$ and $r_{3} \in(-\infty,-1)$.

As in the situation above, the points $\left(0,0, r_{1}\right)$ and $\left(0,0, r_{3}\right)$ are hyperbolic saddles and $\left(0,0, r_{2}\right)$ is a hyperbolic node. This ends the proof of the $H_{2}$ case. 
Case 3: $\Delta_{H}>0$. Then the cubic polynomial $R(p)$ has only one real root and it follows that this root is located in the interval $(-\infty,-1)$ which is a hyperbolic saddle. This ends the proof of the $H_{1}$ case.

To conclude the proof we observe that, in the chart $(u, v, q), q=\frac{d u}{d v}=$ $\frac{1}{p}$, the Lie-Cartan line field,

$$
Y=q \mathcal{G}_{q} \frac{\partial}{\partial x}+\mathcal{G}_{q} \frac{\partial}{\partial y}-\left(q \mathcal{G}_{x}+\mathcal{G}_{y}\right) \frac{\partial}{\partial q}
$$

defined from the implicit differential equation $\mathcal{G}(u, v, q)=0$, is regular, i.e., $Y(0) \neq 0$, provided $c \neq 0$.

The case $\frac{c}{b}<0$ can be analyzed similarly. If $c=0$, the point $(0,0,0)$, in $(x, y, q)$ coordinates, is a hyperbolic singular point and the analysis of the mean curvature configuration is the same as above. Figure 3.2 illustrates the behavior of $X$ near the projective line.
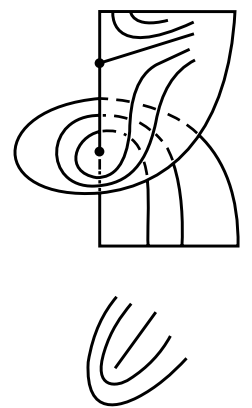
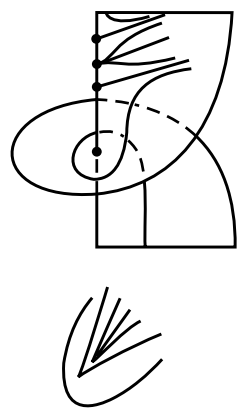
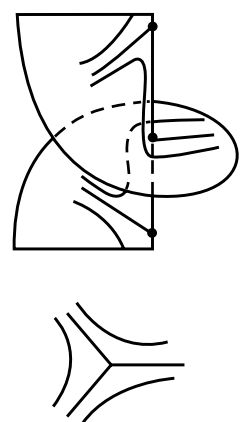

Figure 3.2. Phase portrait of $X$ near the projective line.

Proposition 3.3. In the plane $b=1$ the diagram of the umbilic points of types $H_{i}$ for the mean curvature configuration and of types $D_{i}$ for the principal configuration is as shown in the Figure 3.3.

Proof: The curve $\Delta_{P}=0$ is the union of a parabola with vertex at $(2,0)$ and the line $a=2$, while the curve $\Delta_{H}=0$ is a curve of order four, having two connect components, with a vertex at $(1,0)$ and a cuspidal point at $(5,0)$. The transversal intersection between $\Delta_{P}=0$ and $\Delta_{H}=0$ occurs at the points $(2, \pm \sqrt{9+6 \sqrt{3}})$ and $(11+6 \sqrt{3}, \pm 2(\sqrt{9+6 \sqrt{3}})$. 


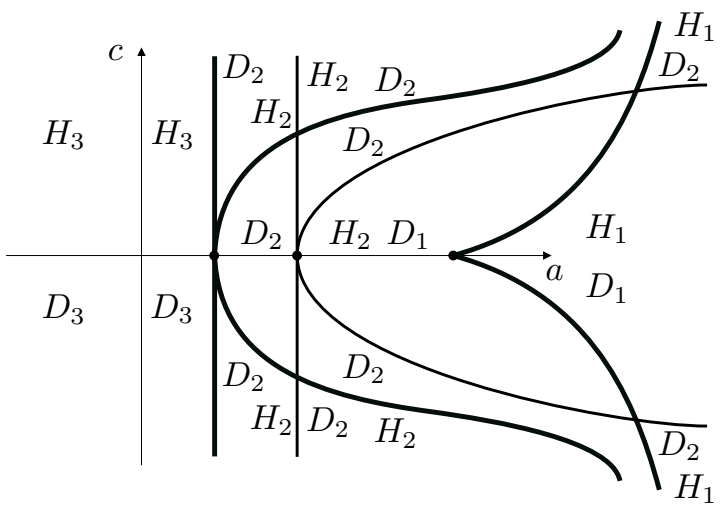

Figure 3.3. Bifurcation diagram of umbilic points $D_{i}$ and $H_{i}$.

Theorem 3.1. An immersion $\alpha \in \mathcal{M}^{k}, k \geq 4$, is $C^{3}$-local mean curvature structurally stable at $\mathcal{U}_{\alpha}$ if only if every $p \in \mathcal{U}_{\alpha}$ is one of the types $H_{i}, i=1,2,3$ of Proposition 3.2.

Proof: Clearly Proposition 3.2 shows that the conditions $H_{i}, i=1,2,3$ $\left(b(b-a) \neq 0\right.$ included) imply the $C^{3}$-local mean curvature structural stability. This involves the construction of the homeomorphism (by means of canonical regions) mapping simultaneously minimal and maximal mean curvature lines around the umbilic points of $\alpha$ onto those of a $C^{4}$ slightly perturbed immersion.

We will discuss the necessity of the transversality condition $(b-a) b \neq$ 0 and of the conditions $H_{i}$. The first one follows from its identification with a transversality condition that guarantees the persistent isolatedness of the umbilic points of $\alpha$ as well as the persistent regularity of the Lie-Cartan surface $\mathcal{G}$. Failure of this condition implies the elimination or splitting of the umbilic point, in contradiction with the local stability assumption. The necessity of $H_{i}$ follows from its dynamic identification with the hyperbolicity of the equilibria along the projective line of the vector field $\mathcal{G}$. Failure of this condition would make possible to change the number of mean curvature umbilic separatrices at the umbilic point by means a small perturbation of the immersion. 


\section{Periodic mean curvature lines}

Let $\alpha: \mathbb{M}^{2} \rightarrow \mathbb{R}^{3}$ be an immersion of a compact and oriented surface and consider the foliations given by the mean curvature lines.

In terms of geometric invariant, here is established an integral expression of the first derivative of the return map of a periodic mean curvature line, called mean curvature cycle. Recall that the return map associated to a cycle is a local diffeomorphism with a fixed point, defined on a cross section normal to the cycle by following the integral curves through this section until they meet again the section. This map is called holonomy in Foliation Theory and Poincaré Map in Dynamical Systems.

A mean curvature cycle is called hyperbolic if the first derivative of the return map at the fixed point is different from one.

The mean curvature configuration $\mathbb{Q}_{\alpha}$ has no mean curvature cycles such that the return map reverses the orientation.

Initially, the integral expression for the derivative of the return map is obtained in class $C^{6}$; see Lemma 4.2 and Proposition 4.1. Later on, in Remark 4.1 it is shown how to extend it to class $C^{3}$.

The characterization of hyperbolicity of mean curvature cycles in terms of local structural stability is given in Theorem 4.1 of this section.

Lemma 4.1. Let $c: I \rightarrow \mathbb{M}^{2}$ be a mean curvature line parametrized by arc length. Then the Darboux frame is given by:

$$
\begin{aligned}
T^{\prime} & =k_{g} N \wedge T+\mathcal{H} N \\
(N \wedge T)^{\prime} & =-k_{g} T+\tau_{g} N \\
N^{\prime} & =-\mathcal{H} T-\tau_{g} N \wedge T
\end{aligned}
$$

where $\tau_{g}= \pm \sqrt{\mathcal{H}^{2}-\mathcal{K}}$. The sign of $\tau_{g}$ is positive (resp. negative) if $c$ is maximal (resp. minimal) mean curvature line.

Proof: The normal curvature $k_{n}$ of the curve $c$ is by the definition the mean curvature $\mathcal{H}$. From the Euler equation $k_{n}=k_{1} \cos ^{2} \theta+k_{2} \sin ^{2} \theta=$ $\mathcal{H}$ if and only if $\theta= \pm \pi / 4$. Therefore the geodesic torsion given by $\tau_{g}=\left(k_{2}-k_{1}\right) \sin \theta \cos \theta= \pm \sqrt{\mathcal{H}^{2}-\mathcal{K}}$. 
Lemma 4.2. Let $\alpha: \mathbb{M} \rightarrow \mathbb{R}^{3}$ be an immersion of class $C^{k}, s \geq k$, and $c$ be a mean curvature cycle of $\alpha$, parametrized by arc length and of length $L$. Then the expression,

$$
\begin{aligned}
\alpha(s, v)=c(s)+v(N & \wedge T)(s) \\
& +\left[\frac{\mathcal{H}(s)}{2} v^{2}+\frac{A(s)}{6} v^{3}+v^{3} B(s, v)\right] N(s)
\end{aligned}
$$

where $B(s, 0)=0$, defines a local chart $(s, v)$ of class $C^{k-6}$ in a neighborhood of c. Moreover $A(s)=2 \mathcal{H}_{v}(s)-\tau_{g}^{\prime}(s)$.

Proof: The curve $c$ is of class $C^{k-1}$ and the map $\alpha(s, v, w)=c(s)+$ $v(N \wedge T)(s)+w N(s)$ is of class $C^{k-2}$ and is a local diffeomorphism in a neighborhood of the axis $s$. In fact $\left[\alpha_{s}, \alpha_{v}, \alpha_{w}\right](s, 0,0)=1$. Therefore there is a function $W(s, v)$ of class $C^{k-2}$ such that $\alpha(s, v, W(s, v))$ is a parametrization of a tubular neighborhood of $\alpha \circ c$. Now for each $s$, $W(s, v)$ is just a parametrization of the curve of intersection between $\alpha(\mathbb{M})$ and the normal plane generated by $\{(N \wedge T)(s), N(s)\}$. This curve of intersection is tangent to $(N \wedge T)(s)$ at $v=0$ and notice that $k_{n}(N \wedge T)(s)=\mathcal{H}(s)$. Therefore,

$$
\begin{aligned}
\alpha(s, v, W(s, v))=c(s)+v & (N \wedge T)(s) \\
+ & {\left[\frac{\mathcal{H}(s)}{2} v^{2}+\frac{A(s)}{6} v^{3}+v^{3} B(s, v)\right] N(s), }
\end{aligned}
$$

where $A$ is of class $C^{k-5}$ and $B(s, 0)=0$.

In the chart $(s, v)$ constructed above it is obtained:

$$
\begin{aligned}
E(s, v) & =1-2 k_{g}(s) v+\text { h.o.t } \\
F(s, v) & =0+0 . v+\text { h.o.t } \\
G(s, v) & =1+0 . v+\text { h.o.t } \\
e(s, v) & =\mathcal{H}(s)+v\left[\tau_{g}^{\prime}(s)-3 k_{g}(s) \mathcal{H}(s)\right]+\text { h.o.t } \\
f(s, v) & =\tau_{g}(s)+\mathcal{H}^{\prime}(s) v+\text { h.o.t } \\
g(s, v) & =\mathcal{H}(s)+v\left[-k_{g}(s) \mathcal{H}(s)+A(s)\right]+\text { h.o.t }
\end{aligned}
$$

where in the expressions above, $E=\left\langle\alpha_{s}, \alpha_{s}\right\rangle, F=\left\langle\alpha_{s}, \alpha_{v}\right\rangle, G=$ $\left\langle\alpha_{v}, \alpha_{v}\right\rangle, e=\left\langle\alpha_{s} \wedge \alpha_{v}, \alpha_{s s}\right\rangle, f=\left\langle\alpha_{s} \wedge \alpha_{v}, \alpha_{s v}\right\rangle$ and $g=\left\langle\alpha_{s} \wedge \alpha_{v}, \alpha_{v v}\right\rangle$.

Now by the definition of mean curvature

$$
\mathcal{H}=\frac{e G+E g-2 f F}{2\left(E G-F^{2}\right)^{3 / 2}},
$$

it follows, by differentiation, that $A(s)=2 \mathcal{H}_{v}-\tau_{g}^{\prime}$. This ends the proof. 
Proposition 4.1. Let $\alpha: \mathbb{M} \rightarrow \mathbb{R}^{3}$ be an immersion of class $C^{k}, k \geq 6$ and $c$ be a mean curvature cycle of $\alpha$, parametrized by arc length $s$ and of total length $L$. Then the derivative of the Poincaré map $\pi_{\alpha}$ associated to $c$ is given by:

$$
\ln \pi_{\alpha}^{\prime}(0)= \pm \frac{1}{2} \int_{0}^{L} \frac{\mathcal{H}_{v} d s}{\sqrt{\mathcal{H}^{2}-\mathcal{K}}}= \pm \frac{1}{2} \int_{c} \frac{\langle N \wedge d c, \nabla H\rangle}{\sqrt{\mathcal{H}^{2}-\mathcal{K}}} .
$$

Proof: The Poincaré map associated to $c$ is the map $\pi_{\alpha}: \Sigma \rightarrow \Sigma$ defined in a transversal section to $c$ such that $\pi_{\alpha}(p)=p$ for $p \in c \cap \Sigma$ and $\pi_{\alpha}(q)$ is the first return of the mean curvature line through $q$ to the section $\Sigma$, choosing a positive orientation for $c$. It is a local diffeomorphism and is defined, in the local chart $(s, v)$ introduced in Lemma 4.2, by $\pi_{\alpha}:\{s=$ $0\} \rightarrow\{s=L\}, \pi_{\alpha}\left(v_{0}\right)=v\left(L, v_{0}\right)$, where $v\left(s, v_{0}\right)$ is the solution of the Cauchy problem

$$
L(s, v) d v^{2}+M(s, v) d s d v+N(s, v) d s^{2}=0, \quad v\left(0, v_{0}\right)=v_{0} .
$$

Here $L(s, v)=g\left(E G-F^{2}\right)+2 f F G-e G^{2}, M(s, v)=4 f E G-2 E F g-$ $2 F G e$ and $N(s, v)=e\left(E G-2 F^{2}\right)+2 E F f-E^{2} g$ and by the equation (4.3) it follows that $L(s, 0)=N(s, 0)=0$ and $M(s, 0)=4 \tau_{g}(s) \neq 0$.

Direct calculation gives that the derivative of the Poincaré map satisfies the following linear differential equation:

$$
\begin{aligned}
\frac{d}{d s}\left(\frac{d v}{d v_{0}}\right) & =-\frac{N_{v}}{M}\left(\frac{d v}{d v_{0}}\right) \\
& =-\frac{\left[e\left(E G-2 F^{2}\right)+2 E F f-E^{2} g\right]_{v}}{[4 f E G-2 E F g-2 F G e]}\left(\frac{d v}{d v_{0}}\right) .
\end{aligned}
$$

Therefore, using equation (4.3) and that $A(s)=2 \mathcal{H}_{v}-\tau_{g}^{\prime}$ it results that

(4.6) $\frac{d}{d s}\left(\left.\frac{d v}{d v_{0}}\right|_{v_{0}=0}\right)=-\frac{1}{4 \tau_{g}}\left(\tau_{g}^{\prime}-A\right)\left(\frac{d v}{d v_{0}}\right)=\left(-\frac{\tau_{g}^{\prime}}{2 \tau_{g}}+\frac{\mathcal{H}_{v}}{2 \tau_{g}}\right) \frac{d v}{d v_{0}}$.

Performing the integration of the equation (4.6) along an arc $\left[s_{0}, s_{1}\right]$ of mean curvature line it follows that,

$$
\left.\frac{d v}{d v_{0}}\right|_{v_{0}=0}=\frac{\left(\tau_{g}\left(s_{1}\right)\right)^{\frac{-1}{2}}}{\left(\tau_{g}\left(s_{0}\right)\right)^{\frac{-1}{2}}} \exp \left[ \pm \frac{1}{2} \int_{s_{0}}^{s_{1}} \frac{\mathcal{H}_{v}}{\sqrt{\mathcal{H}^{2}-\mathcal{K}}} d s\right]
$$

Here we have used that $\mathcal{K}=\left(e g-f^{2}\right) /\left(E G-F^{2}\right)^{2}$ and therefore at $v=0 \mathcal{K}=\mathcal{H}^{2}-\tau_{g}^{2}$. 
Applying (4.7) along the mean curvature cycle of length $L$, obtain

$$
\ln \left(\pi_{\alpha}^{\prime}(0)=\ln \left(\left.\frac{d v}{d v_{0}}\right|_{v_{0}=0}\right)= \pm \frac{1}{2} \int_{0}^{L} \frac{\mathcal{H}_{v}}{\sqrt{\mathcal{H}^{2}-\mathcal{K}}} d s\right.
$$

This ends the proof.

Remark 4.1. At this point we show how to extend the expression for the derivative of the hyperbolicity of mean curvature cycles established for class $C^{6}$ to class $C^{3}$ (in fact we need only class $C^{4}$ ).

The expression (4.7) is the derivative of the transition map for a mean curvature foliation (which at this point is only of class $C^{1}$ ), along an arc of mean curvature line. In fact, this follows by approximating the $C^{3}$ immersion by one of class $C^{6}$. The corresponding transition map (now of class $C^{4}$ ) whose derivative is given by expression (4.7) converges to the original one (in class $C^{1}$ ) whose expression must given by the same integral, since the functions involved there are the uniform limits of the corresponding ones for the approximating immersion.

Remark 4.2. The expression for the derivative of the Poincaré map is obtained by the integration of a one form along the mean curvature line $\gamma$. In the case of the principal lines the correspondent expression for the derivative is given by: $\ln \pi^{\prime}(0)=\frac{1}{2} \int_{\gamma} \frac{d \mathcal{H}}{\sqrt{\mathcal{H}^{2}-\mathcal{K}}}$. This was proved in $[\mathbf{S}-\mathbf{G}],[\mathbf{G S 2}]$.

Proposition 4.2. Let $\alpha: \mathbb{M} \rightarrow \mathbb{R}^{3}$ be an immersion of class $C^{k}, k \geq 6$ and $c$ be a maximal mean curvature cycle of $\alpha$, parametrized by arc length and of length L. Consider a chart $(s, v)$ as in Lemma 4.2 and consider the deformation

$$
\beta_{\epsilon}(s, v)=\beta(\epsilon, s, v)=\alpha(s, v)+\epsilon\left[\frac{a(s)}{6} v^{3}\right] \delta(v) N(s)
$$

where $\delta=1$ in neighborhood of $v=0$, with small support and $a>0$.

Then $c$ is a mean curvature cycle of $\beta_{\epsilon}$ for all $\epsilon$ small and $c$ is a hyperbolic mean curvature cycle for $\beta_{\epsilon}, \epsilon \neq 0$. 
Proof: In the chart $(s, v)$, for the immersion $\beta$, it is obtained that:

$$
\begin{aligned}
E(s, v) & =1-2 k_{g}(s) v+\text { h.o.t } \\
F(s, v) & =0+0 . v+\text { h.o.t } \\
G(s, v) & =1+0 . v+\text { h.o.t } \\
e(s, v) & =\mathcal{H}(s)+v\left[\tau_{g}^{\prime}(s)-3 k_{g}(s) \mathcal{H}(s)\right]+\text { h.o.t } \\
f(s, v) & =\tau_{g}(s)+\mathcal{H}^{\prime}(s) v+\text { h.o.t } \\
g(s, v) & =\mathcal{H}(s)+v\left[-k_{g}(s) \mathcal{H}(s)+A(s)+\epsilon a(s)\right]+\text { h.o.t } .
\end{aligned}
$$

In the expressions above $E=\left\langle\beta_{s}, \beta_{s}\right\rangle, F=\left\langle\beta_{s}, \beta_{v}\right\rangle, G=\left\langle\beta_{v}, \beta_{v}\right\rangle$, $e=\left\langle\beta_{s s}, \beta_{s} \wedge \beta_{v}\right\rangle, f=\left\langle\beta_{s} \wedge \beta_{v}, \beta_{s v}\right\rangle$ and $g=\left\langle\beta_{s} \wedge \beta_{v}, \beta_{v v}\right\rangle$.

Therefore $c$ is a maximal mean curvature cycle for all $\beta_{\epsilon}$ and at $v=0$ it follows that $2 \mathcal{H}_{v}=A+\epsilon a-\tau_{g}^{\prime}$.

Therefore, assuming $a(s)>0$, it results that,

$$
\left.\frac{d}{d \epsilon}\left(\ln \pi^{\prime}(0)\right)\right|_{\epsilon=0}= \pm \frac{1}{2} \int_{0}^{L} \frac{a}{\sqrt{\mathcal{H}^{2}-\mathcal{K}}} \neq 0 .
$$

As a synthesis of Propositions 4.1 and 4.2, we obtain the following.

Theorem 4.1. An immersion $\alpha \in \mathcal{M}^{k}, k \geq 4$, is $C^{3}$-local mean curvature structurally stable at a mean curvature cycle $c$ if only if,

$$
\int_{c} \frac{\langle N \wedge d c, \nabla H\rangle}{\sqrt{\mathcal{H}^{2}-\mathcal{K}}} \neq 0
$$

Proof: Standard.

\section{Examples of mean curvature configurations}

In this section is made a preliminary comparative study of Principal and Mean curvature configurations. Also, two examples about the global behavior of mean curvature lines will be analyzed.

\subsection{Analogy and discrepancy with two classical results.}

Proposition 5.1. Let $I: \mathbb{R}^{3}-\{0\} \rightarrow \mathbb{R}^{3}-\{0\}$ be the inversion with respect to the unitary sphere $\mathbb{S}^{2}, I(x, y, z)=\frac{1}{x^{2}+y^{2}+z^{2}}(x, y, z)=\frac{1}{r^{2}}(x, y, z)$. Then the umbilics, mean curvature and the principal curvature lines are of a surface $\mathbb{M}$ are mapped in the same by the inversion, exchanging minimal and maximal in both cases.

Proof: As the inversion is a conformal map and the principal directions are preserved, it follows that the mean curvature directions are also preserved. 
Proposition 5.2. Let $\alpha$ be an immersion of a surface $\mathbb{M}$ into $\mathbb{R}^{3}$ and consider the displacement $\alpha_{\epsilon}=\alpha+\epsilon N_{\alpha}$, where $N_{\alpha}$ is the normal map of $\alpha$. Then the principal lines are preserved along $\alpha_{\epsilon}$ while the mean curvature lines are not. In fact, the mean curvature lines are locally rotated by a nonzero angle.

Proof: Let $(u, v)$ be a principal chart on the surface $\mathbb{M}$.

The coefficients of the fundamental forms of $\alpha$ and of $\alpha_{\epsilon}$ in the principal chart $(u, v)$ are related by:

$$
\begin{aligned}
\bar{E} & =\left(1-\epsilon k_{1}\right)^{2} E, & & \bar{e}=\left(1-\epsilon k_{1}\right) e \\
\bar{F} & =F=0, & & \bar{f}=f=0 \\
\bar{G} & =\left(1-\epsilon k_{2}\right)^{2} G, & & \bar{g}=\left(1-\epsilon k_{2}\right) g .
\end{aligned}
$$

So, the differential equation of mean curvature lines for the immersion $\alpha_{\epsilon}$ is given by:

$$
\begin{aligned}
& \left(1-\epsilon k_{1}\right)^{2} E d u^{2}-\left(1-\epsilon k_{2}\right)^{2} G d v^{2}=0 . \text { Therefore } \frac{d v}{d u}= \pm \sqrt{\frac{E}{G} \frac{1-\epsilon k_{1}}{1-\epsilon k_{2}} .} \\
& \text { It follows that }\left.\frac{d}{d \epsilon}\left(\frac{d v}{d u}\right)\right|_{\{\epsilon=0\}}= \pm \sqrt{\frac{E}{G}}\left(k_{2}-k_{1}\right) \neq 0 .
\end{aligned}
$$

\subsection{Mean curvature lines on the torus of revolution.}

Proposition 5.3. Consider a torus of revolution $T(r, R)$ obtained by rotating a circle of radius $r$ about a line in the same plane and at a distance $R, R>r$, from its center. Define the function

$$
\rho=\rho\left(\frac{R}{r}\right)=\frac{1}{2 \pi} \int_{0}^{2 \pi} \frac{d s}{\frac{R}{r}+\cos (s)} .
$$

Then the mean curvature lines on $T(r, R)$ are all closed or all recurrent according to $\rho \in \mathbb{Q}$ or $\rho \in \mathbb{R} \backslash \mathbb{Q}$ and both cases occur.

Proof: The torus of revolution $T(r, R)$ is parametrized by

$$
\alpha(s, \theta)=((R+r \cos (s)) \cos (\theta),(R+r \cos (s)) \sin (\theta), r \sin (s)) .
$$

Direct calculation shows that $E=r^{2}, F=0, G=[R+r \cos (s)]^{2}$ and $f=0$. Clearly $(s, \theta)$ is a principal chart.

The differential equation of the mean curvature lines, in the principal chart $(s, \theta)$, is given by $E(s, \theta) d s^{2}-G(s, \theta) d \theta^{2}=0$. This is equivalent to $r^{2} d s^{2}=[R+r \cos (s)]^{2} d \theta^{2}$.

Solving the equation above it is follows that,

$$
\theta(2 \pi)=\theta_{0} \pm 2 \pi \rho\left(\frac{R}{r}\right)=\theta_{0} \pm \int_{0}^{2 \pi} \frac{d s}{\frac{R}{r}+\cos (s)} .
$$


So the two Poincaré maps, $\pi_{ \pm}:\{s=0\} \rightarrow\{s=2 \pi\}$, defined by $\pi_{ \pm}\left(\theta_{0}\right)=\theta_{0} \pm 2 \pi \rho\left(\frac{R}{r}\right)$ have rotation number equal to $\pm \rho$. The function $\rho\left(\frac{R}{r}\right)$ is strictly decreasing and its image is the interval $(0, \infty)$, both the rational and irrational cases occur. This ends the proof.

Remark 5.1. All the principal curvature lines are closed in the torus of revolution $T(r, R)$.

\subsection{Mean curvature lines on the ellipsoid.}

Proposition 5.4. Consider an ellipsoid $\mathbb{E}_{a, b, c}$ with three axes $a>b>$ $c>0$. Then $\mathbb{E}_{a, b, c}$ have four umbilic points located in the plane of symmetry orthogonal to middle axis; they are of the type $H_{1}$ for mean curvature lines and of type $D_{1}$ for the principal curvature lines.

Proof: Without lost of generality suppose that $\mathbb{E}_{a, b, c}$ is defined by the equation $\frac{x^{2}}{a^{2}}+\frac{y^{2}}{b^{2}}+\frac{z^{2}}{c^{2}}=1$, with $c=1$, and write $A=\frac{1}{a^{2}}$ and $B=\frac{1}{b^{2}}$. Consider the parametrization of the ellipsoid $\alpha(x, y)=(x, y, h(x, y))=$ $\left(x, y, \sqrt{1-A x^{2}-B y^{2}}\right)$. Calculation shows that:

$$
\begin{array}{rlrl}
E=1+\left(\frac{A x}{h}\right)^{2} & F=\frac{A B x y}{h^{2}} & G=1+\left(\frac{B y}{h}\right)^{2} \\
e=-\frac{A h^{2}-A^{2} x^{2}}{h^{3}} & f=-\frac{A B x y}{h} & g=-\frac{B h^{2}-B^{2} y^{2}}{h^{3}} .
\end{array}
$$

Therefore it is obtained that:

$$
\begin{aligned}
L(x, y) & =(F g-G f)(x, y)=\frac{A B(1-B) x y}{h^{3}} \\
M(x, y) & =(E g-G e)(x, y)=\frac{(A-B)+A B(1-A) x^{2}+A B(B-1) y^{2}}{h^{3}} \\
N(x, y) & =(E f-F e)(x, y)=\frac{A B(A-1) x y}{h^{3}} .
\end{aligned}
$$

As $A<B<1$ it follows that the four umbilic points are:

$$
\left( \pm x_{0}, 0, \pm z_{0}\right)=\left( \pm \sqrt{\frac{B-A}{A B(1-A)}}, 0, \pm \sqrt{\frac{A(1-B)}{B(1-A)}}\right) .
$$


In a neighborhood of the umbilic point $\left(x_{0}, 0, z_{0}\right)$ it follows that the first order jet of the differential equation $L d y^{2}+M d x d y+N d x^{2}=0$ of principal curvature lines is given by:

$$
\begin{aligned}
{\left[A B(1-B) \frac{x_{0}}{z_{0}^{3}} y\right] d y^{2}+[2 A B(1-A)} & \left.\frac{x_{0}}{z_{0}^{3}}\left(x-x_{0}\right)\right] d x d y \\
& +\left[A B(A-1) \frac{x_{0}}{z_{0}^{3}} y\right] d x^{2}=0
\end{aligned}
$$

Performing a change of coordinates $x=x_{0}+\bar{x}, y=\sqrt{\frac{1-A}{1-B}} \bar{y}$ the following equation is obtained:

$$
(1-A) \sqrt{\frac{1-A}{1-B}}\left[\bar{y}(d \bar{y})^{2}+2 \bar{x} d \bar{x} d \bar{y}-\bar{y}(d \bar{x})^{2}\right]=0 .
$$

Therefore by Proposition 3.1 this umbilic point is of type $D_{1}$ and the same holds for the all the other umbilic points.

In a neighborhood of the umbilic point $\left(x_{0}, 0, z_{0}\right)$ the first order jet of the differential equation of the mean curvature lines is given by:

$$
\left[(1-A)\left(x-x_{0}\right)\right] d y^{2}+[2(A-1) y] d x d y+\left[-\frac{(A-1)^{2}}{1-B}\left(x-x_{0}\right)\right] d x^{2}=0 .
$$

In the differential equation above perform the change of coordinates $x=x_{0}+\bar{x}, y=\sqrt{\frac{1-A}{1-B}} \bar{y}$ to obtain:

$$
\frac{(1-A)^{2}}{1-B}\left[\bar{x}(d \bar{y})^{2}-2 \bar{y} d \bar{x} d \bar{y}-\bar{x}(d \bar{x})^{2}\right]=0 .
$$

Therefore by Proposition 3.2 the umbilic point is of type $H_{1}$ for the mean curvature lines.

Proposition 5.5. Consider an ellipsoid $\mathbb{E}_{a, b, c}$ with three axes $a>b>$ $c>0$. On the ellipse $\Sigma \subset \mathbb{E}_{a, b, c}$, containing the four umbilic points, $p_{i}, i=1, \ldots, 4$, counterclockwise oriented, denote by $s_{1}$ (resp. $s_{2}$ ) the elliptic distance between the adjacent umbilic points $p_{1}$ and $p_{4}$ (resp. $p_{1}$ and $\left.p_{2}\right)$. Define $\rho=\frac{s_{2}}{s_{1}}$.

Then if $\rho \in \mathbb{R} \backslash \mathbb{Q}$ (resp. $\rho \in \mathbb{Q}$ ) all the mean curvature lines are recurrent (resp. all, with the exception of the mean curvature umbilic separatrices, are closed). See Figure 5.1. 


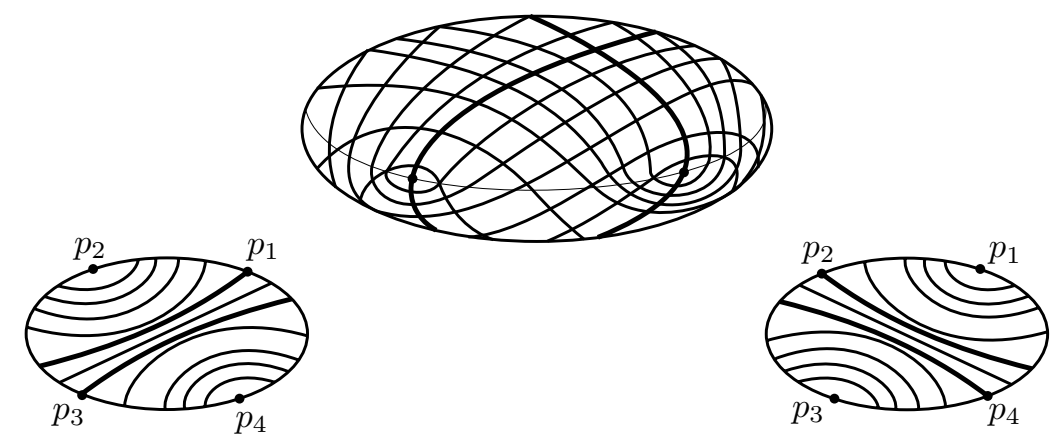

Figure 5.1. Mean curvature lines on the ellipsoid.

Proof: The ellipsoid $\mathbb{E}_{a, b, c}$ belongs to the triple orthogonal system of surfaces defined by the one parameter family of quadrics, $\frac{x^{2}}{a^{2}+\lambda}+\frac{y^{2}}{b^{2}+\lambda}+$ $\frac{z^{2}}{c^{2}+\lambda}=1$ with $a>b>c>0$, see also [St] and $[\mathbf{S p}]$.

The following parametrization of $\mathbb{E}_{a, b, c}$

$$
\begin{array}{r}
\alpha(u, v)=\left( \pm \sqrt{\frac{a^{2}\left(u+a^{2}\right)\left(v+a^{2}\right)}{\left(b^{2}-a^{2}\right)\left(c^{2}-a^{2}\right)}}, \pm \sqrt{\frac{b^{2}\left(u+b^{2}\right)\left(v+b^{2}\right)}{\left(b^{2}-a^{2}\right)\left(b^{2}-c^{2}\right)}},\right. \\
\left. \pm \sqrt{\frac{c^{2}\left(u+c^{2}\right)\left(v+c^{2}\right)}{\left(c^{2}-a^{2}\right)\left(c^{2}-b^{2}\right)}}\right)
\end{array}
$$

defines the ellipsoidal coordinates $(u, v)$ on $\mathbb{E}_{a, b, c}$, where $u \in\left(-b^{2},-c^{2}\right)$ and $v \in\left(-a^{2},-b^{2}\right)$.

The first fundamental form of $\mathbb{E}_{a, b, c}$ is given by:

$$
\begin{aligned}
& d s^{2}=E d u^{2}+G d v^{2}=\frac{1}{4} \frac{(u-v) u}{\left(u+a^{2}\right)\left(u+b^{2}\right)\left(u+c^{2}\right)} d u^{2} \\
& +\frac{1}{4} \frac{(v-u) v}{\left(v+a^{2}\right)\left(v+b^{2}\right)\left(v+c^{2}\right)} d v^{2} .
\end{aligned}
$$

The four umbilic points are $\left( \pm x_{0}, 0, \pm z_{0}\right)=\left( \pm a \sqrt{\frac{a^{2}-b^{2}}{a^{2}-c^{2}}}, 0, \pm c \sqrt{\frac{c^{2}-b^{2}}{c^{2}-a^{2}}}\right)$. 
On the ellipse $\Sigma=\left\{(x, 0, z) \mid\left\{\frac{x^{2}}{a^{2}}+\frac{z^{2}}{c^{2}}=1\right\}\right.$ the distance between the umbilic points $p_{1}=\left(x_{0}, 0, z_{0}\right)$ and $p_{4}=\left(x_{0}, 0,-z_{0}\right)$ is given by $s_{1}=$ $\int_{-b^{2}}^{-c^{2}} \frac{\sqrt{u}}{\left(u+a^{2}\right)\left(u+c^{2}\right)} d u$ and that between the umbilic points $p_{1}=\left(x_{0}, 0, z_{0}\right)$ and $p_{2}=\left(-x_{0}, 0, z_{0}\right)$ is given by $s_{2}=\int_{-a^{2}}^{-b^{2}} \frac{\sqrt{v}}{\left(v+a^{2}\right)\left(v+c^{2}\right)} d v$.

It is obvious that the ellipse $\Sigma$ is the union of four umbilic points and four principal umbilical separatrices for the principal foliations. So $\Sigma \backslash\left\{p_{1}, p_{2}, p_{3}, p_{4}\right\}$ is a transversal section of both mean curvature foliations. The differential equation of the mean curvature lines in the principal chart $(u, v)$ is given by $E d u^{2}-G d v^{2}=0$, which is equivalent to $(\sqrt{E} d u)^{2}=(\sqrt{G} d v)^{2}$, which amounts to $d s_{1}= \pm d s_{2}$. Therefore near the umbilic point $p_{1}$ the mean curvature lines with a mean curvature umbilic separatrix contained in the region $\{y>0\}$ define a the return map $\sigma_{+}: \Sigma \rightarrow \Sigma$ which is an isometry, reverting the orientation, with $\sigma_{+}\left(p_{1}\right)=p_{1}$. This follows because in the principal chart $(u, v)$ this return map is defined by $\sigma_{+}:\left\{u=-b^{2}\right\} \rightarrow\left\{v=-b^{2}\right\}$ which satisfies the differential equation $\frac{d s_{2}}{d s_{1}}=-1$. By analytic continuation it results that $\sigma_{+}$is a isometry reverting orientation with two fixed points $\left\{p_{1}, p_{3}\right\}$. The geometric reflection $\sigma_{-}$, defined in the region $y<0$ have the two umbilic $\left\{p_{2}, p_{4}\right\}$ as fixed points.

So the Poincaré return map $\pi_{1}: \Sigma \rightarrow \Sigma$ (composition of two isometries $\sigma_{+}$and $\left.\sigma_{-}\right)$is a rotation with rotation number given by $\frac{s_{2}}{s_{1}}$.

Analogously for the other mean curvature configuration, with the Poincaré return map given by $\pi_{2}=\tau_{+} \circ \tau_{-}$where $\tau_{+}$and $\tau_{-}$are two isometries having respectively $\left\{p_{2}, p_{4}\right\}$ and $\left\{p_{1}, p_{3}\right\}$ as fixed points.

\section{Structural stability of mean curvature configurations}

Let $\mathbb{M}^{2}$ be a compact, smooth and oriented surface. Denote by $\mathcal{M}^{k, s}$ be the space of $C^{k}$ immersions of $\mathbb{M}$ into the Euclidean space $\mathbb{R}^{3}$, endowed with the $C^{s}$ topology. Consider the subset $\mathcal{Q}^{k}$ of immersions $\alpha$ defined by the following conditions:

a) all umbilic points are of types $H_{1}, H_{2}$ or $H_{3}$ for mean curvature lines;

b) all mean curvature cycles are hyperbolic;

c) the limit set of every mean curvature line is contained in the set of umbilic points and mean curvature cycles of $\alpha$;

d) all mean curvature umbilic separatrices are associated to a single umbilic point; this means that there are no connections or self 
connections of mean curvature umbilic separatrices of both mean curvature configurations.

Theorem 6.1. Let $k \geq 4$. The following holds:

i) The subset $\mathcal{Q}^{k}$ is open in $\mathcal{M}^{k, 3}$.

ii) Every $\alpha \in \mathcal{Q}^{k}$ is Mean Curvature Structurally Stable.

Proof: The openness of $\mathcal{Q}^{k}$ it follows from the local structure of the mean curvature lines near the umbilic points $H_{i}, i=1,2,3$, near the mean curvature cycles and by the absence of umbilic mean curvature separatrix connections and the absence of recurrences. The equivalence can be performed by the method of canonical regions and their continuation as was done in $[\mathbf{S}-\mathbf{G}],[\mathbf{G S 2}]$, for principal lines, and $[\mathbf{G a}-\mathbf{G u}-\mathbf{S}]$, for asymptotic lines.

\section{Density of mean curvature structurally stable immersions}

In this section will be proved an approximation theorem for the class of immersions or surfaces having structurally stable mean curvature configuration.

Theorem 7.1. Let $k \geq 4$. The subset $\mathcal{Q}^{k}$ is dense in $\mathcal{M}^{k, 2}$.

The proof of this theorem follows from the elimination of mean curvature recurrences and the stabilization of the mean curvature umbilical separatrices. The steps are basically those followed by C. Gutiérrez and J. Sotomayor in the case of principal curvature lines, see [GS1], [GS2]. The main ideas goes back to M. Peixoto $[\mathbf{P e}]$ and C. Pugh $[\mathbf{P u}]$ to solve the similar problem of elimination of recurrences for vector fields on surfaces. See also the book by J. Palis and W. de Melo $[\mathbf{P}-\mathbf{M}]$.

It will be established in what follows the main ingredients of the proof of the Approximation Theorem, with the complete proofs of the preliminary technical lemmas necessary to obtain the Lifting Lemma, essential to control the mean curvature lines under suitable deformation of the immersion. There is lost of generality to assume that the immersion is $C^{\infty}$ or $C^{\omega}$ in the proof of the density theorem.

In what follows a chart whose coordinates lines are mean curvature lines will be called mean curvature chart for $\alpha$. 
Lemma 7.1. Let $\alpha: \mathbb{M} \rightarrow \mathbb{R}^{3}$ be an immersion of class $C^{\infty}$ and $(u, v)$ : $(U, D) \rightarrow(V, I \times I)$ be a positive mean curvature chart on $\mathbb{M}$, where $I=[-1,1]$. Suppose that, for $\epsilon$ small, $\beta=\alpha_{\epsilon}=\alpha+\epsilon \varphi N$ is an immersion and $\varphi$ be a smooth function on $U$ which satisfies: $\varphi(-1, v)=$ $\varphi(1, v)=\varphi_{u}(-1, v)=\varphi_{u}(1, v)=\varphi_{u u}(-1, v)=\varphi_{u u}(1, v)=0$. Then the mean curvature line of $\alpha_{\epsilon}$ on $D$ which passes through $q$ in $\{u=$ $-1\} \cap\{-1<v<1\}$ meets the segment of abscissa $\{u=1\}$ at a point whose $v$-coordinate $v_{\epsilon}$ has a derivative with respect to $\epsilon$ given by:

$$
\begin{aligned}
\left.\frac{d}{d \epsilon}\left(v_{\epsilon}\right)\right|_{\epsilon=0}= & \int_{-1}^{1} \frac{E}{4 \sqrt{E G} \sqrt{\mathcal{H}^{2}-\mathcal{K}}} \varphi_{v v} d u-\int_{-1}^{1} \frac{(E G)_{v}}{8 f G^{2}} \varphi_{v} d u \\
& +\int_{-1}^{1}\left[\frac{f}{G}-\left(\frac{1}{4 f}\right)_{u u}-\left(\frac{\left(\ln (\sqrt{E G})_{u}\right.}{4 f}\right)_{u}\right] \varphi d u
\end{aligned}
$$

Proof: Suppose that for $\epsilon$ small,

$\beta(u, v, \epsilon)=\alpha_{\epsilon}(u, v)=\alpha(u, v)+\epsilon \varphi(u, v) N(u, v)$ is an immersion.

The $v$-coordinate, $v=v(u, q, \epsilon)$, of the point where the line of mean curvature through the point $q$ in $\{u=-1\} \cap\{-1<v<1\}$ meets the curve with abscissa $\{u\}$, satisfies the following Cauchy Problem with parameter $\epsilon$.

$$
\begin{aligned}
& {\left[e\left(E G-2 F^{2}\right)+2 E F f-E^{2} g\right]+[4 f E G-2 E F g-2 F G e] \frac{d v}{d u}} \\
& \quad+\left[g\left(E G-2 F^{2}\right)+2 f F G-e G^{2}\right]\left(\frac{d v}{d u}\right)^{2}=0, \quad v(-1, \epsilon)=q .
\end{aligned}
$$

Since $(u, v)$ is a mean curvature chart it results that

$$
\begin{aligned}
\frac{d v}{d u}(u, q, 0) & =0, \\
F(u, v, 0) & =0, \\
\mathcal{H}(u, v, 0) & =\frac{e}{E}(u, v, 0)=\frac{g}{G}(u, v, 0) .
\end{aligned}
$$


Lines of Mean Curvature on Surfaces Immersed in $\mathbb{R}^{3} 453$

Differentiating the equation (7.2) with respect to $\epsilon$, evaluated on $(u, v(q), \epsilon)$, making $\epsilon=0$ and using (7.3) it follows that $\frac{d v_{\epsilon}}{d \epsilon}=\left.\frac{\partial v_{\epsilon}}{\partial \epsilon}(u, q, \epsilon)\right|_{\epsilon=0}$ satisfies the following Cauchy Problem:

$$
\begin{aligned}
& \frac{d}{d u}\left(\frac{d v_{\epsilon}}{d \epsilon}\right)=\frac{\left[e_{\epsilon}(E G)+e(E G)_{\epsilon}+2 f E F_{\epsilon}-g_{\epsilon} E^{2}+2 g E E_{\epsilon}\right]}{[4 f E G]}(u, v(q), 0) \\
& \frac{d v_{\epsilon}}{d \epsilon}(-1, q, 0)=0
\end{aligned}
$$

The structure equations for the immersion $\alpha$ are given by:

$$
\begin{aligned}
N_{u} & =\frac{f F-e G}{E G-F^{2}} \alpha_{u}+\frac{e F-f E}{E G-F^{2}} \alpha_{v} \\
N_{v} & =\frac{g F-f G}{E G-F^{2}} \alpha_{u}+\frac{f F-g E}{E G-F^{2}} \alpha_{v} \\
\alpha_{u u} & =\Gamma_{11}^{1} \alpha_{u}+\Gamma_{11}^{2} \alpha_{v}+e N \\
\alpha_{u v} & =\Gamma_{12}^{1} \alpha_{u}+\Gamma_{12}^{2} \alpha_{v}+f N \\
\alpha_{v v} & =\Gamma_{22}^{1} \alpha_{u}+\Gamma_{22}^{2} \alpha_{v}+g N .
\end{aligned}
$$

The functions $\Gamma_{i j}^{k}$ are the Christoffel symbols whose expression in terms of $E$ and $G$ in a mean curvature chart are given by:

$$
\begin{array}{lll}
\Gamma_{11}^{1}=\frac{E_{u}}{2 E}, & \Gamma_{11}^{2}=-\frac{E_{v}}{2 G}, & \Gamma_{12}^{1}=\frac{E_{v}}{2 E} \\
\Gamma_{12}^{2}=\frac{G_{u}}{2 G}, & \Gamma_{22}^{1}=-\frac{G_{u}}{2 E}, & \Gamma_{22}^{2}=\frac{G_{v}}{2 G} .
\end{array}
$$

By direct calculation, it is obtained

$$
\begin{aligned}
& \beta_{u}=\left(1-\epsilon \varphi \frac{e}{E}\right) \alpha_{u}-\epsilon \varphi \frac{f}{G} \alpha_{v}+\epsilon \varphi_{u} N \\
& \beta_{v}=-\epsilon \varphi \frac{f}{E} \alpha_{u}+\left(1-\epsilon \varphi \frac{g}{G}\right) \alpha_{v}+\epsilon \varphi_{v} N
\end{aligned}
$$


454

R. Garcia, J. Sotomayor

$$
\begin{aligned}
\beta_{u u}= & {\left[-\epsilon(\varphi \mathcal{H})_{u}+\Gamma_{11}^{1}(1-\epsilon \varphi \mathcal{H})-\epsilon \varphi \frac{f}{G} \Gamma_{12}^{1}-\epsilon \varphi_{u} \mathcal{H}\right] \alpha_{u} } \\
& +\left[(1-\epsilon \varphi \mathcal{H}) \Gamma_{11}^{2}-\epsilon\left(\varphi \frac{f}{G}\right)_{u}-\epsilon \varphi \frac{f}{G} \Gamma_{12}^{2}-\epsilon \varphi_{u} \frac{f}{G}\right] \alpha_{v} \\
& +\left[(1-\epsilon \varphi \mathcal{H}) e-\epsilon \varphi \frac{f^{2}}{G}+\epsilon \varphi_{u u}\right] N \\
\beta_{u v}= & {\left[-\epsilon(\varphi \mathcal{H})_{v}+(1-\epsilon \varphi \mathcal{H}) \Gamma_{12}^{1}-\epsilon \varphi \frac{f}{G} \Gamma_{22}^{1}-\epsilon \varphi_{u} \frac{f}{E}\right] \alpha_{u} } \\
& +\left[(1-\epsilon \varphi \mathcal{H}) \Gamma_{12}^{2}-\epsilon\left(\varphi \frac{f}{G}\right)_{v}-\epsilon\left(\varphi \frac{f}{G}\right) \Gamma_{22}^{2}-\epsilon \varphi_{u} \mathcal{H}\right] \alpha_{v} \\
& +\left[(1-2 \epsilon \varphi \mathcal{H}) f+\epsilon \varphi{ }_{u v}\right] N \\
\beta_{v v}= & {\left[-\epsilon\left(\varphi \frac{f}{E}\right)_{v}-\epsilon \varphi \frac{f}{E} \Gamma_{12}^{1}+(1-\epsilon \varphi \mathcal{H}) \Gamma_{22}^{1}-\epsilon \varphi_{v} \frac{f}{E}\right] \alpha_{u} } \\
& +\left[-\epsilon \varphi \frac{f}{E} \Gamma_{12}^{2}-\epsilon(\varphi \mathcal{H})_{v}+(1-\epsilon \varphi \mathcal{H}) \Gamma_{22}^{2}-\epsilon \varphi_{v} \mathcal{H}\right] \alpha_{v} \\
+ & {\left[-\epsilon \varphi \frac{f^{2}}{E}+(1-\epsilon \varphi \mathcal{H}) g+\epsilon \varphi_{v v}\right] N }
\end{aligned}
$$

Also,

$$
\left.\frac{\partial}{\partial \epsilon}\left(\beta_{u} \wedge \beta_{v}\right)\right|_{\epsilon=0}=-2 \varphi \mathcal{H} \alpha_{u} \wedge \alpha_{v}+\varphi_{u} N \wedge \alpha_{v}+\varphi_{v} \alpha_{u} \wedge N .
$$

Therefore, using the equations (7.7)-(7.10) the following is obtained.

$$
\begin{gathered}
E_{\epsilon}=-2 \varphi e, \quad F_{\epsilon}=-2 \varphi f, \quad G_{\epsilon}=-2 \varphi g \\
e_{\epsilon}=\left[\varphi_{u u}-3 \varphi e \mathcal{H}-\varphi_{u} \Gamma_{11}^{1}-\varphi_{v} \Gamma_{11}^{2}-\varphi \frac{f^{2}}{G}\right]\left\langle N, \alpha_{u} \wedge \alpha_{v}\right\rangle \\
g_{\epsilon}=\left(\varphi_{v v}-3 \varphi \mathcal{H} g-\varphi \frac{f^{2}}{E}-\varphi_{u} \Gamma_{22}^{1}-\varphi_{v} \Gamma_{22}^{2}\right)\left\langle N, \alpha_{u} \wedge \alpha_{v}\right\rangle .
\end{gathered}
$$

Let $N=e\left(E G-2 F^{2}\right)+2 f E F-g E^{2}$ and $M=4 f E G-2 g E F-2 e F G$.

Then, using (7.6) and (7.12), it follows that

$$
\left.\frac{N_{\epsilon}}{M}\right|_{\epsilon=0}=\frac{\varphi_{u u}}{4 f}-\frac{\varphi_{v v} E}{4 f G}-\frac{\varphi_{u}(E G)_{u}}{8 f E G}+\frac{(E G)_{v} \varphi_{v}}{8 f G^{2}}-\frac{f \varphi}{G} .
$$




\section{Lines of Mean Curvature on Surfaces Immersed in $\mathbb{R}^{3} 455$}

Using (7.13) when integrating the variational equation (7.4) and performing the partial integration with boundary conditions on the function $\varphi$, is achieved the expression for $\left.\left(\frac{d v_{\epsilon}}{d \epsilon}\right)\right|_{\epsilon=0}$ as stated in (7.1).

Lemma 7.2. Let $\alpha: \mathbb{M} \rightarrow \mathbb{R}^{3}$ be an immersion of class $C^{\infty}$ and $(u, v)$ : $(U, D) \rightarrow(V, I \times I)$ be a positive mean curvature chart on $\mathbb{M}$, where $I=[-1,1]$. Then there exists a smooth function $\varphi: \mathbb{M} \rightarrow[0,1]$ whose support is contained in $D$ such that, if $\epsilon$ is small enough then, for every $\epsilon$ in $[-r, r], \beta=\alpha+\epsilon \varphi N$ is an immersion and the mean curvature line for $\beta$ on $D$ which passes through $q$ in $\{u=-1\} \cap\{-1<v<1\}$ meets the segment $\{u=1\} \times\{-1<v<1\}$ at a point $v_{\epsilon}(q)$ so that the map $\epsilon \rightarrow v_{\epsilon}(q)$ is strictly increasing.

Proof: Let $m$ be a real smooth function with values in $[0,1]$, identically equal to 1 on a neighborhood of 0 and with support contained in $I$.

Let $\varphi=\varphi(u, v)=b \frac{v^{2}}{2} m(u) m(v)$ and take $r>0$ small so that for any $\epsilon$ in $[-r, r], \beta=\alpha_{\epsilon}=\alpha+\epsilon \varphi N$ is a smooth immersion. Let $v(u)$, $u \in I$, be the $v$-coordinate of the mean curvature lines of $\alpha_{\epsilon}=\alpha+\epsilon \varphi N$ such that $v_{\epsilon}(q)=q$. As $\varphi(u, 0)=\varphi_{v}(u, 0)=0$ and $\varphi_{v v}(u, 0)=b m(u)$ by Lemma 7.1 applied to the family of immersions $\alpha_{\epsilon}$ it follows that

$$
\left.\frac{\partial v}{\partial \epsilon}(u, \epsilon)\right|_{(0,0)}=\int_{-1}^{1} \frac{E}{4 \sqrt{E G} \sqrt{\mathcal{H}^{2}-\mathcal{K}}} m(u) d u=c>0 .
$$

This implies that the map $\epsilon \rightarrow v_{\epsilon}(q)$ is strictly increasing. This proves the lemma.

Lemma 7.3. Let $\alpha: \mathbb{M} \rightarrow \mathbb{R}^{3}$ be an immersion and $(u, v):(U, D) \rightarrow$ $(V, I \times I)$ be a positive mean curvature chart for $\alpha$ on $\mathbb{M}$, where $I=$ $[-1,1]$. Then given any $\eta>0$, there are numbers $d, c \in\left(0, \frac{1}{12}\right)$ such that for every $r \in(0, d]$ and $q$ in $\{u=-1\} \cap\left\{-\frac{1}{2}<v<\frac{1}{2}\right\}$, there exists a smooth function $\varphi: \mathbb{M} \rightarrow[0,1]$ whose support is contained in $D_{r}=v^{-1}(v(q)+r I)$ and $\|\varphi\|_{2, V}$, the $C^{2}$-norm of $\varphi$ on $V$, in the $(u, v)$-coordinate chart, is less than $\eta$.

Furthermore, for every $\epsilon \in I, \alpha_{\epsilon}=\alpha+\epsilon \varphi N$ is an immersion and the mean curvature line for $\alpha_{\epsilon}$ on $D$ which passes through $q$ in $\{u=$ $-1\} \cap\{-1<v<1\}$ meets the segment $\{u=1\} \cap\{-1<v<1\}$ at a point $v_{\epsilon}(q)$ so that the map $\epsilon \rightarrow v_{\epsilon}(q)$ is strictly increasing and its image contains the interval $[v(q)-2 c \epsilon, v(q)+2 c \epsilon]$. 
Proof: Let $m$ be a real smooth function with values in $[0,1]$, identically equal to 1 on $\frac{5}{6} I$ and with support contained in $\frac{6}{7} I$. Let also $\eta>0$ be given. There are real numbers $c>0$ and $b$ such that for all $\left(u_{0}, v_{0}\right)$ in $I \times I$ it follows that,

$$
\begin{aligned}
6|b|\left(\|m\|_{2}\right)^{2} & <\eta \\
\int_{-1}^{0} b m(u) \frac{E}{4 \sqrt{E G} \sqrt{\mathcal{H}^{2}-\mathcal{K}}}\left(u, v_{0}\right) d u & <\frac{1}{4} \\
\int_{-1}^{1} b m(u) \frac{E}{4 \sqrt{E G} \sqrt{\mathcal{H}^{2}-\mathcal{K}}}\left(u, v_{0}\right) d u & >3 c .
\end{aligned}
$$

Let $\psi$ be a smooth real function on $U \times I \times I$, defined by

$$
\psi\left(u, v ; v_{0}, \epsilon\right)=b \epsilon \frac{\left(v-v_{0}\right)^{2}}{2} m(u) .
$$

It will be proved that if $d=d(\eta) \in\left(0, \frac{1}{12}\right)$ is small enough, then for every $\epsilon \in(0, d)$ and $q$ with $u(q)=-1$ and $v(q)=v_{0}$ in $\frac{1}{2} I$, the smooth function $\varphi()=.\varphi(. ; v(q), \epsilon)$ defined on $\mathbb{M}$ by

$$
\varphi\left(u, v ; v_{0}, \epsilon\right)=\psi\left(u, v ; v_{0}, \epsilon\right) m\left(\frac{v-v_{0}}{|\epsilon|}\right)
$$

whose support is contained in $D_{r}$, satisfies the conditions required by the lemma.

In fact, suppose $d>0$ is so small that for any $\left(v_{0}, \epsilon\right) \in I \times d I$, $\alpha_{v_{0}, \epsilon}=\alpha+\psi\left(. ; v_{0}, \epsilon\right) N$ is an immersion.

Let $v\left(u ; v_{0}, \epsilon\right), u \in I$ and $v \in \frac{4}{5} I$, be the $v$-coordinate of a mean curvature line of $\alpha_{\epsilon}$ through the point $q$, with $u(q)=-1$ and $v(q)=v_{0}$. As $\psi\left(u, v_{0} ; v_{0}, \epsilon\right)=0$, using (7.14a), (7.14b) and (7.14c), it follows from Lemmas 7.1 and 7.2, applied to the family of immersions $\alpha_{v_{0}, \epsilon}$, depending on the parameter $\epsilon$, that for all $\left(u, v_{0}\right)$ in $I \times \frac{3}{4} I$,

$$
\frac{\partial v}{\partial \epsilon}\left(1, v_{0}, 0\right)>2 c \text { and } \frac{\partial v}{\partial \epsilon}\left(1, v_{0}, 0\right)<\frac{1}{3} .
$$

Hence, as $I$ is compact and $\frac{\partial v}{\partial \epsilon}\left(1, v_{0}, \epsilon\right)$ depends continuously on $\left(v_{0}, \epsilon\right)$, taking $d>0$ small enough, it holds that for all $\left(u ; v_{0}, \epsilon\right)$ in $I \times \frac{1}{2} I \times d I$,

$$
\frac{\partial v}{\partial \epsilon}\left(1, v_{0}, 0\right)>c \quad \text { and } \quad \frac{\partial v}{\partial \epsilon}\left(1, v_{0}, 0\right)<\frac{1}{2} .
$$


Therefore from the Mean Value Theorem, for all $\left(u ; v_{0}, \epsilon\right)$ in $I \times \frac{1}{2} I \times d I$

$$
\begin{array}{ll}
v\left(1 ; v_{0}, \epsilon\right) \geq v_{0}+c \epsilon, & \text { if } \epsilon \leq 0 \\
v\left(1 ; v_{0}, \epsilon\right) \leq v_{0}+c \epsilon, & \text { if } \epsilon<0 \quad \text { and } \\
\left|v\left(u ; v_{0}, \epsilon\right)-v_{0}\right| \leq \frac{|\epsilon|}{2} . &
\end{array}
$$

Without lost of generality, $\eta>0$ can be assumed to be so small that for $\epsilon \in I, \alpha_{\epsilon}=\alpha+\epsilon \varphi N$ is an immersion. Notice that for all $v$ in $v(q)+\frac{5}{6} \epsilon I, m\left(\frac{v-v_{0}}{|\epsilon|}\right)=1$. Therefore for all $\epsilon$ in $I$ and all $(u, v)$ in $I \times\left(v(q)+\frac{5}{6} \epsilon I\right), \varphi\left(u, v ; v_{0}, \epsilon\right)=\psi\left(u, v ; v_{0}, \epsilon\right)$. From this and $(7.15 \mathrm{c})$ follows that the mean curvature lines of $\left.\left(\alpha+\psi\left(., v_{0}, \epsilon\right) N\right)\right|_{D^{\prime}}$ and those of the $\alpha+\left.\varphi\left(., v_{0}, \epsilon\right) N\right|_{D^{\prime}}$ coincide, where $D^{\prime}=v^{-1}\left[v(q)+\frac{5}{6} \epsilon I\right]$. Hence, the assertion that the map $v_{\epsilon}(q)$ is strictly increasing and its image contains the interval $[v(q)-2 c \epsilon, v(q)+2 c \epsilon]$ follows from (7.15a) and (7.15b).

Lemma 7.4 (Lifting Lemma). Let $\alpha: \mathbb{M} \rightarrow \mathbb{R}^{3}$ be an immersion of class $C^{\infty}$ with a minimal mean curvature line $\tilde{\gamma}$, oriented from a starting point $q$, whose $\omega$-limit set contains a nontrivial minimal recurrent mean curvature line $\gamma$. Then given any $\eta>0, p \in \gamma$ and any mean curvature chart $(u, v):(U, D, p) \rightarrow(V, I \times I, 0)$ where $I=[-1,1]$, there is a mean curvature chart $(s, t): D^{\prime} \rightarrow I \times I$ for $\alpha$ on $\mathbb{M}$ and a smooth function $\varphi: \mathbb{M} \rightarrow[0,1]$ such that:

i) The support of $\varphi$ is contained in $D \cap D^{\prime}$ and $\|\varphi\|_{2, V}$, the $C^{2}$-norm of $\varphi$ on $V$, is less than $\eta$.

ii) There are arcs of minimal mean curvature lines $[b, a] \subset[q, a] \subset \tilde{\gamma}$ such that $a, b$ are in the arc $\{s=-1\}$ and $[b, a] \cap D^{\prime}=[q, a] \cap D^{\prime} \subset$ $\left[a, a^{\prime}\right] \cup\left[b, b^{\prime}\right]$, where $a^{\prime}$ and $b^{\prime}$ are the points on $\{u=1\}$, defined by $t\left(a^{\prime}\right)=t(a)$ and $t\left(b^{\prime}\right)=t(b)$.

Moreover the minimal mean curvature lines for $\alpha_{\epsilon}$ on $D^{\prime}$ which passes through a (resp. b) meets the segment $\{u=1\}$ at a point $v_{\epsilon}(a)$ (resp. $v_{\epsilon}(b)$ ) in such way that for some values of $\epsilon \in[0,1]$, it coincides with $a^{\prime}$ and $b^{\prime}$. See Figure 7.1 .

Proof: See [GS1], [GS2]. 
Proposition 7.1. Let $\alpha: \mathbb{M} \rightarrow \mathbb{R}^{3}$ be an immersion of class $C^{\infty}$ with umbilic set $\mathcal{U}_{\alpha} \neq \emptyset$ and having a nontrivial minimal recurrent mean curvature line $\gamma$ and let $A$ be a subset of $\mathbb{M}$ formed by finitely many minimal mean curvature lines that are either minimal mean curvature umbilical connections or minimal mean curvature cycles. Then there is a point $p \in \gamma \backslash A$ such that given any chart $(u, v):(U, p) \rightarrow(V, 0)$ on a neighborhood $U$ of $p$, where $U$ is disjoint of $A$, there is a sequence of smooth functions $\varphi_{n}$ on $\mathbb{M}$, whose support is contained in $U$ such that $\left\|\varphi_{n}\right\|_{2, V}$, the $C^{2}$-norm of $\varphi_{n}$, in the coordinate chart $(u, v)$, tends to 0 and such that the immersions $\alpha_{n}=\alpha+\varphi_{n} N$ satisfy the following alternatives:

i) $\alpha_{n}$ has a mean curvature cycle $\gamma_{n}$ not completely contained in $\mathbb{M} \backslash U$. Moreover if there is a minimal mean curvature cycle of $\alpha$ (i.e. disjoint of $U$ ) which together with $\gamma_{n}$ bound a cylinder in $\mathbb{M}$ then this cylinder contains an umbilic point of $\alpha$.

ii) $\alpha_{n}$ has at least one minimal mean curvature umbilical connection more than the immersion $\alpha$ does.

Proof: See [GS1], [GS2].

Proposition 7.2. Let $\alpha: \mathbb{M} \rightarrow \mathbb{R}^{3}$ be an immersion of class $C^{\infty}$ and let $A$ be a subset of $\mathbb{M}$ formed by finitely many minimal mean curvature lines that are either minimal mean curvature umbilical connections or minimal mean curvature cycles.

Then there is a sequence of immersions $\alpha_{n}=\alpha+\varphi_{n} N, C^{2}$-converging to $\alpha$, such that the support of $\varphi_{n}$ is disjoint from $\bar{A}=A \cup \mathcal{U}_{\alpha}$ and $\alpha_{n}$ has no non trivial minimal recurrent mean curvature lines.

Proof: See [GS1], [GS2].

Let $\alpha: \mathbb{M} \rightarrow \mathbb{R}^{3}$ be an immersion whose umbilic points are of type $H_{i}$, $i=1,2,3$. A minimal mean curvature umbilic separatrix $\Gamma$ of an umbilic point $p$ is said to be stabilized provided:

i) it is not a minimal mean curvature umbilical connection for $\alpha$;

ii) its limit sets are umbilic points or attracting or repelling minimal mean curvature cycles, and

iii) $\alpha$ is in the $C^{3}$-interior of the set of immersions that satisfy i) and ii); i.e., for any sequence of immersions $\alpha_{n}, C^{3}$-converging to $\alpha_{n}$, the sequence of separatrices $\Gamma_{n}$, of an umbilic point $p_{n}$, converging to the separatrix $\Gamma$ of $p$, verify i) and ii) for $\alpha_{n}$. 
Lines of Mean Curvature on Surfaces Immersed in $\mathbb{R}^{3} 459$

Lemma 7.5 (Stabilization Lemma). Any immersion $\alpha: \mathbb{M} \rightarrow \mathbb{R}^{3}$ of class $C^{\infty}$ is the $C^{2}$-limit of a sequence of immersions whose umbilic points are all of type $H_{i}, i=1,2,3$ and furthermore:

i) their minimal mean curvature umbilical separatrices are all stabilized;

ii) the $\omega$-limit set of any oriented minimal mean curvature line is either an umbilic point or a minimal mean curvature cycle, and

iii) for any $s \geq 3, \alpha$ is in the $C^{s}$-interior of the set of immersions satisfying i) and ii).

Proof: See [GS1], [GS2].

Remark 7.1. In all lemmas and propositions above the same conclusions hold for the maximal mean curvature lines provided the corresponding hypotheses are made also in this case.

\subsection{Proof of the Approximation Theorem 7.1.}

Part 1: Elimination of nontrivial recurrences:

By Proposition 7.2 the recurrent lines can always be destroyed by a finite sequence of small local $C^{2}$-perturbations of the immersion $\alpha$. Each perturbation creates either a new mean curvature cycle or a new mean curvature umbilical connection.

Initially will be considered the elimination of the minimal recurrent mean curvature lines.

The key points involved in the argument will be given below.

Let $\gamma$ be a non trivial minimal recurrent mean curvature line.

Assume first that $\gamma$ is orientable, i.e., it is possible to give an orientation in $\gamma$ such that on a mean curvature chart it is induced by an orientation defined locally on the mean curvature line field by the chart. The recurrent lines on vector fields and those of mean curvature foliations on the Torus, in Section 5, are of this type. In this case there is a piecewise smooth simple closed curve of the form $[\underline{b}, \underline{a}] \cup[\underline{b} ; \underline{a}]$, with $[a, b] \subset \gamma$ and $\underline{a}$ near $\underline{b}$, that can be slightly perturbed to obtain a minimal mean curvature cycle for the approximating immersion. Here, and in what follows, $[\underline{b} ; \underline{a}]$ means an arc of a maximal mean curvature line and $[\underline{b}, \underline{a}]$ is an arc of a minimal mean curvature line. The arrangement of these points are illustrated in Figure 7.1.a). 


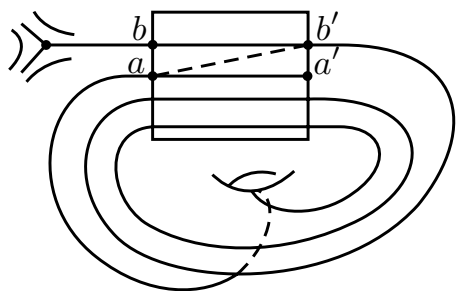

a)

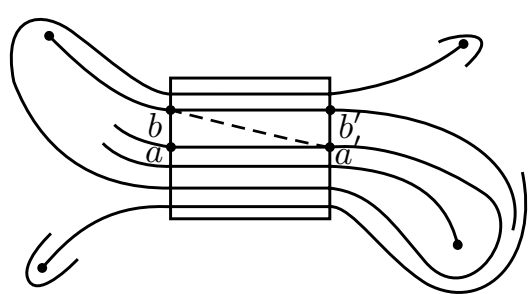

b)

Figure 7.1. Recurrences of mean curvature lines.

When the recurrence is oscillatory (i.e. non-orientable), then there is no such simple closed curve available. In this case there are minimal mean curvature umbilical separatrices accumulating on $p$. These separatrices can be connected by means of a small perturbation of the immersion. The mean curvature lines on the ellipsoid, Section 5, presents this type of recurrence. This situation is illustrated in Figure 7.1.b).

The possibility of finding perturbations as those described above is established in the Lifting Lemma 7.4 and Proposition 7.1.

This is done as follows.

Consider a non trivial recurrent minimal umbilical separatrix $\gamma^{\prime}$ of an umbilic point $q$. Take $p \in \gamma$ and a mean curvature chart $(u, v):(D, p) \rightarrow$ $(I \times I, 0)$.

By Lemma 7.1, arbitrarily close to $\{v=0\}$, two points $\underline{a}, \underline{b}$ in $\{u=$ $-1\} \cap \gamma^{\prime}$ can be selected such that $v(\underline{b})-v(\underline{a})=2 r>0$ and $(\underline{b}, \underline{a})$ has the following spacing property relatively to the maximal mean curvature arc $[\underline{a} ; \underline{b}]$ :

$(\underline{b}, \underline{a})$ is disjoint from $\{v(\underline{a})-(3 / 2) r<v<v(\underline{b})+(3 / 2) r\} \cap\{u=-1\}$.

It results from this that a local version of the lifting argument (Lemma 7.2) can be applied to obtain, by means of an $\epsilon$-small $C^{2}$-perturbation supported on $\{v(\underline{a})-(3 / 2) r<v<v(\underline{b})+(3 / 2) r\}$, the following. Given $\eta>0$, there is a constant $c=c(\eta,(u, v))>0$, which does not depend on how close $\underline{a}$ and $\underline{b}$ are, such that, for every $\underline{x} \in[\underline{a} ; \underline{b}]$, the mean curvature line through the point $\underline{x}$ can reach any point of the segment $\{u=1\} \cap$ $\{v(\underline{x})-2 c r<v<v(\underline{x})+2 c r\}$.

Consider first the assumption that $c=1$.

If $[\underline{b}, \underline{a}] \backslash\left\{v(\underline{a})-\frac{3 r}{2}<v<v(\underline{b})+\frac{3 r}{2}\right\}=\left(\underline{b}^{\prime}, \underline{a}\right)$, with $\underline{b}^{\prime} \in\{u=1\}$, then, via a perturbation, a minimal mean curvature cycle can be obtained. This is illustrated in Figure 7.1.a). 
If, however, $[\underline{b}, \underline{a}] \backslash\left\{v(\underline{a})-\frac{3 r}{2}<v<v(\underline{b})+\frac{3 r}{2}\right\}=\left(\underline{b}^{\prime}, \underline{a}^{\prime}\right)$ with $\underline{b}^{\prime}, \underline{a}^{\prime} \in$ $\{u=1\}$, it seems to be difficult to approximate $\left[\underline{b}^{\prime}, \underline{a}^{\prime}\right]$ by a minimal mean curvature cycle. Nevertheless, by moving $\underline{a}^{\prime}$ towards $\underline{b}^{\prime}$, one can generate a continuous family of minimal mean curvature arcs with endpoints in $\{u=1\}$. In this process the resulting endpoints become close to each other but cannot coincide. Using this it is proved that the limit set of this family of minimal mean curvature arcs must contain an umbilic point $\underline{q}^{\prime}$ and an arc $\left[\underline{p}^{\prime}, \underline{q}^{\prime}\right]$, of a minimal mean curvature umbilical separatrix of $\underline{q}^{\prime}$, intersecting

$$
\left\{v(\underline{a})-\frac{3 r}{2}<v<v(\underline{b})+\frac{3 r}{2}\right\} \cap\{u=1\}
$$

at a point of $\left(\underline{b}^{\prime} ; \underline{a}^{\prime}\right)$. In this situation, via a perturbation, a minimal mean curvature umbilical connection between $\underline{q}$ and $\underline{q}^{\prime}$ can be produced. This is illustrated in Figure 7.1.b).

In general, $c>0$ is much smaller than 1 and the analysis is done by showing that Lemma 7.2 can be used a number $n$ of times, where $n$ is of the order of $1 / c$, to finally obtain enough lifting as to make possible the application of the arguments above. The $n$ intervals $\left[\underline{a}_{i} ; \underline{b}_{i}\right]$ which play the same role as that performed by $[\underline{a} ; \underline{b}]$ and on which Lemma 7.2 is to be used, are described below.

If $\underline{a}$ and $\underline{b}$ are close enough to each other and $(\underline{a}, \underline{b})$ is long enough, it is proved that there is a family $\left\{\left[\underline{a}_{t} ; \underline{b}_{t}\right] ; t \in[1, n]\right\}$ of pairwise disjoint maximal mean curvature arcs such that:

i) $\left[\underline{a}_{1} ; \underline{b}_{1}\right]=[\underline{a} ; \underline{b}]$,

ii) the curves $\underline{a}_{t} \in \tilde{\gamma}, \underline{b}_{t} \in \tilde{\gamma}$ are regular,

iii) for all $i \in\{1,2, \ldots, n\},\left[\underline{a}_{i} ; \underline{b}_{i}\right]$ is contained in $\{u= \pm 1\}$, and $\left(\underline{b}_{i}, \underline{a}_{i}\right) \backslash\left\{v=v\left(\underline{b}_{i}\right), v\left(\underline{a}_{i}\right)\right\}$ is disjoint from $D_{i}$. Here $D_{i}=\left\{v\left(\underline{a}_{i}\right)-\right.$ $\left.r_{i}<v<v\left(\underline{b}_{i}\right)+r_{i}\right\} \subset\left\{-\frac{1}{2}<v<\frac{1}{2}\right\}$ with $2 r_{i}=\left|v\left(\underline{b}_{i}\right)-v\left(\underline{a}_{i}\right)\right|$ and, finally,

iv) the sets $D_{i}, i \in\{1,2, \ldots, n\}$, are pairwise disjoint.

See Figure 7.2.a) keeping in mind that $D_{i}$ is the sub rectangle of $D$ with vertical edges $\left\{\tilde{s}=x_{i}\right\}$ and $\left\{\tilde{s}=x_{i}^{\prime}\right\}$. See also Figure 7.2.b) keeping in mind that $D_{i}$ is the rectangle with vertices $\underline{z}_{i}^{\prime}, \underline{z}_{i}, \underline{w}_{i}^{\prime}$, and $\underline{w}_{i}$.

By Lemma 7.2 the amount of lifting gained in each set $D_{i}$ is $2 c$ times $r_{i}$ and it is carried to $\left[\underline{a}_{i+1} ; \underline{b}_{i+1}\right]$, rescaled almost linearly, by the mean curvature foliation. Consequently, as $n c$ is near 1 , all of these lifting can be added up as required.

So, all recurrent minimal mean curvature lines can be eliminated. 
To eliminate the recurrent maximal mean curvature lines of $\mathbb{Q}_{\alpha, 2}$, it is necessary to perform the same deformation analysis as above, applied to this case, with no fundamental change.

The mean curvature umbilical separatrices of $\mathbb{Q}_{\alpha, 1}$ are stabilized taking care to consider the $C^{2}$-deformations of the immersion $\alpha$, with support in mean curvature charts disjoint from the nowhere dense set $A$ (see Proposition 7.2) consisting of the minimal mean curvature umbilical separatrices and the minimal mean curvature cycles. So the stabilized minimal mean curvature umbilical separatrices and minimal mean curvature cycles are preserved and these deformations do not produce any new non trivial recurrence for the minimal mean curvature lines.

Therefore the immersion $\alpha$ can be approximated in the $C^{2}$-topology by an immersion $\alpha_{1}$ having all minimal and maximal mean curvature umbilical separatrices stabilized.

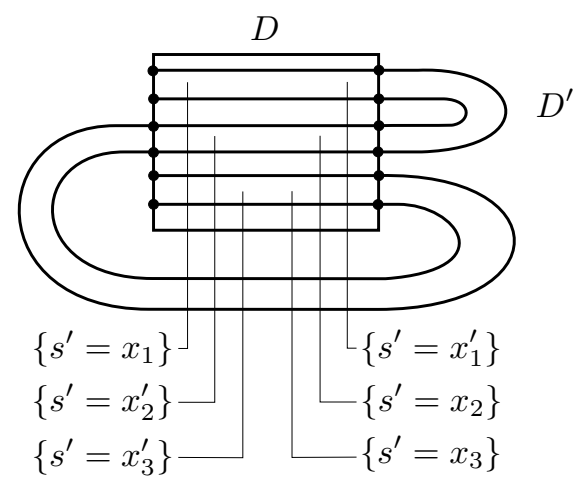

a)

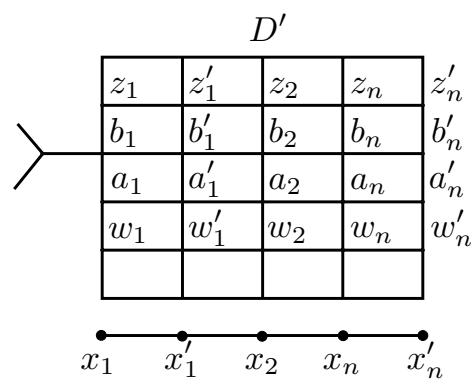

b)

FiguRE 7.2. Lifting of mean curvature lines.

Part 2: Conclusion of the Proof of Theorem 7.1:

The first step is to approximate an immersion $\alpha$ of compact and oriented surface $\mathbb{M}$ by an immersion having all umbilic points of the type $H_{i}$, $i=1,2,3$. This can be done by the Transversality Theorem establishing the condition $T=b(b-a) \neq 0$ and by a finite number of small 
local changes on the coefficients of the third jet of the immersion at the umbilic points.

Next approximate the immersion $\alpha$ in $C^{s}$-sense by an analytic immersion, which will be denoted by $\alpha_{1}$. There are two cases to consider.

Case 1: The surface $\mathbb{M}$ is diffeomorphic to a torus and the immersion $\alpha_{1}$ is without umbilics.

By using Proposition 7.2 it is possible to obtain an analytic immersion $\alpha^{\prime}, C^{2}$ close to $\alpha_{1}$ having only finitely many mean curvature cycles, all of which have finite multiplicity.

The resulting immersion $\alpha^{\prime}$ can be deformed around a mean curvature cycle to a obtain an immersion with a hyperbolic mean curvature cycle. If this immersion is approximated by an analytic one, $\alpha^{\prime \prime}$, will have only finitely many mean curvature cycles, all of which with finite multiplicity. In either case, using Proposition $4.2, \alpha^{\prime \prime}$ can be approximated by an immersion $\tilde{\alpha}$, all whose mean curvature cycles are hyperbolic, which belong to the class $\mathcal{Q}^{k}$, since conditions i), iii) and iv) are guaranteed by the Stabilization Lemma 7.4. This ends the proof in this case.

Case 2: The analytic immersion $\alpha_{1}$ have umbilic points and all are of the types $H_{i}, i=1,2,3$.

In this case, as shown in Part 1 , the immersion $\alpha_{1}$ can be taken so that both, minimal and maximal mean curvature umbilical separatrices are stabilized and without non trivial recurrences. The next step, using Proposition 4.2, is to deform the immersion in order to obtain an immersion with all minimal and maximal mean curvature cycles hyperbolic. This ends the proof.

\section{Further developments and concluding remarks}

Let $\alpha: \mathbb{M} \rightarrow \mathbb{R}^{3}$ be an immersion of class $C^{k}, k \geq 4$. The principal configuration of $\alpha$ is the triple $\mathbb{P}_{\alpha}=\left\{\mathcal{U}_{\alpha}, \mathbb{P}_{\alpha, 1}, \mathbb{P}_{\alpha, 2}\right\}$, where $\mathbb{P}_{\alpha, i}, i=1,2$, are respectively the minimal and maximal principal foliations of $\alpha$.

Denote by $\mathcal{P}^{k}$ the class of immersions which are principal structurally stable. The main result on this subject was established in $[\mathbf{S}-\mathbf{G}]$, [GS1], [GS2], where the following theorem was proved.

Theorem 8.1 ([S-G], [GS1], [GS2]). For $k \geq 4$ the subset $\mathcal{P}^{k}$ is open in $\mathcal{M}^{k, 3}$ and dense in $\mathcal{M}^{k, 2}$.

Therefore from Theorems 6.1, 7.1 and 8.1 the following will be established. 
Theorem 8.2. The subset $\mathcal{Q}^{k} \cap \mathcal{P}^{k}$ is open in $\mathcal{M}^{k, 3}$ and dense in $\mathcal{M}^{k, 2}$ for $k \geq 4$.

Proof: The openness is clear. To prove the density take an immersion $\alpha \in \mathcal{P}^{k}$. Assume that the umbilic points of $\alpha$ are simultaneously of types $D_{i}$ and $H_{i}, i=1,2,3$, see Proposition 3.3. Approximate $\alpha$ in the $C^{2}$ topology by an immersion in $\mathcal{Q}^{k}$, taking care so that the perturbations be localized away from the umbilic points, principal cycles and principal umbilic separatrices as in Section 7.

Remark 8.1. Theorem 8.2 implies an important complement to a previous paper of the authors concerning axial configurations of immersions into $\mathbb{R}^{4}$. See the Introduction above and [Ga-So1]. Let $\alpha: \mathbb{T}^{2} \rightarrow \mathbb{R}^{3}$ be an immersion of class $C^{k}, k \geq 4$, of the torus $\mathbb{T}^{2}$ such that $\alpha \in \mathcal{P}^{k} \cap \mathcal{Q}^{k}$ and $\mathcal{U}_{\alpha}=\emptyset$. Then $\alpha$ satisfies the conditions for principal and mean axial structurally stability given by Theorem 6.1 in [Ga-So1]. Therefore the class of immersions of the torus in $\mathbb{R}^{4}$ which are principal and mean axial stable is open and not empty in $\mathcal{M}^{k}$.

Remark 8.2. The main point of the forthcoming paper, [Ga-So2], is to modify the analysis above for the torus to the case of immersions $\alpha \in$ $\mathcal{P}^{k} \cap \mathcal{Q}^{k}$ with $\mathcal{U}_{\alpha} \neq \emptyset$ to obtain immersions in $\mathbb{R}^{4}$ which are principal and mean axial stable.

\section{References}

[B-F] J. W. Bruce And D. L. Fidal, On binary differential equations and umbilics, Proc. Roy. Soc. Edinburgh Sect. A 111(1-2) (1989), 147-168.

[Da] G. DARBoux, "Leçons sur la théorie générale des surfaces. Vol. IV: Sur la forme des lignes de courbure dans la voisinage d'un ombilic". Note 07, Gauthier Villars, Paris, 1896.

[Ga-Gu-S] R. Garcia, C. Gutiérrez and J. Sotomayor, Structural stability of asymptotic lines on surfaces immersed in $\mathbb{R}^{3}$, Bull. Sci. Math. 123(8) (1999), 599-622.

[Ga-M-F-R] R. Garcia, D. K. H. Mochida, M. D. C. RomeRo Fuster and M. A. S. Ruas, Inflection points and topology of surfaces in 4-space, Trans. Amer. Math. Soc. 352(7) (2000), 3029-3043.

[Ga-So1] R. Garcia and J. Sotomayor, Lines of axial curvature on surfaces immersed in $\mathbb{R}^{4}$, Differential Geom. Appl. 12(3) (2000), 253-269. 
[Ga-So2] R. Garcia and J. Sotomayor, A class of axial structurally stable immersions of surfaces into $\mathbb{R}^{4}$, in preparation.

[G-G-T-G] C. Gutiérrez, I. Guadalupe, R. Tribuzy and V. Guí$\tilde{N} E Z$, Lines of curvature on surfaces immersed in $\mathbb{R}^{4}$, Bol. Soc. Brasil. Mat. (N.S.) 28(2) (1997), 233-251.

[GS1] C. GutiérRez and J. Sotomayor, An approximation theorem for immersions with stable configurations of lines of principal curvature, in "Geometric dynamics" (Rio de Janeiro, 1981), Lecture Notes in Math. 1007, Springer, Berlin, 1983, pp. 332-368.

[GS2] C. GutiérRez and J. Sotomayor, Lines of curvature and umbilical points, in "Structurally Stable Configurations of Lines of Curvature and Umbilic Points on Surfaces", Monografias del IMCA, Lima, Peru, 1998, IMPA, Rio de Janeiro, Brazil, 1991, pp. 1-84.

[GS3] C. GutiérRez And J. Sotomayor, Lines of curvature, umbilic points and Carathéodory conjecture, Resenhas 3(3) (1998), 291-322.

[Gu $\quad$ V. Gúí̃̃ez, Positive quadratic differential forms and foliations with singularities on surfaces, Trans. Amer. Math. Soc. 309(2) (1988), 477-502.

[Lit] J. A. LitTLe, On singularities of submanifolds of higher dimensional Euclidean spaces, Ann. Mat. Pura Appl. (4) 83 (1969), 261-335.

[P-M] J. Palis and W. De Melo, "Geometric theory of dynamical systems", Springer-Verlag, New York, 1982.

[Pe] M. M. Peiхото, Structural stability on two-dimensional manifolds, Topology 1 (1962), 101-120.

[Pu $\quad$ C. C. Pugh, The closing lemma, Amer. J. Math. 89 (1967), 956-1009.

[S-G] J. Sotomayor and C. Gutiérrez, Structurally stable configurations of lines of principal curvature, in "Bifurcation, Ergodic theory and applications" (Dijon, 1981), Astérisque 98-99, Soc. Math. France, Paris, 1982, pp. 195215.

[Sp] M. SPIVAK, "A comprehensive introduction to differential geometry. Vol. III", second ed., Publish or Perish Inc., Wilmington, Del., 1979. 
[SX1] B. Smyth And F. Xavier, A sharp geometric estimate for the index of an umbilic on a smooth surface, Bull. London Math. Soc. 24(2) (1992), 176-180.

[SX2] B. Smyth And F. Xavier, Real solvability of the equation $\partial \frac{2}{z} \omega=\rho g$ and the topology of isolated umbilics, J. Geom. Anal. 8(4) (1998), 655-671.

[St] D. J. Struik, "Lectures on classical differential geometry", second ed., Dover Publications Inc., New York, 1988.

Ronaldo Garcia:

Instituto de Matemática e Estatística

Universidade Federal de Goiás

C. Postal 131, Goiânia, GO

CEP 74001-970

Brazil

E-mail address: ragarcia@mat.ufg.br

Jorge Sotomayor:

Instituto de Matemática e Estatística

Universidade de São Paulo

C. Postal 66281, São Paulo, SP

CEP 05315-970

Brazil

E-mail address: sotp@ime.usp.br

Primera versió rebuda el 24 d'octubre de 2000 , darrera versió rebuda el 9 de març de 2001. 